\title{
Marked Effect of Aromatic Solvent on Unfolding Rate of Helical Ethynylhelicene Oligomer
}

\author{
Hiroki Sugiura, Yasuko Nigorikawa, Yuto Saiki, Keiichi Nakamura, and Masahiko Yamaguchi* \\ Department of Organic Chemistry, Graduate School of Pharmaceutical Sciences, Tohoku University
}

Aoba, Sendai 980-8578, Japan

\section{Supporting Information}

\section{Experimental Section}

Melting points were determined with a Yanaco micro melting point apparatus without correction. Elemental analyses were conducted with a Yanaco CHN CORDER MT-6. Optical rotations were measured on a JASCO DIP-340 digital polarimeter. IR spectra were measured on a JASCO FT/IR-400 spectrophotometer. UV-vis spectra were measured on a BECKMAN DU 640 or a JASCO J-720 spectropolarimeter. ${ }^{1} \mathrm{H}$ NMR and ${ }^{13} \mathrm{C}$ NMR spectra were recorded on a JEOL JNM-ECA600 (600 MHz) with tetramethylsilane as an internal standard. ${ }^{13} \mathrm{C}$ NMR spectra taken in $\mathrm{CDCl}_{3}(\delta 77.0)$ were referenced to the residual solvents. FAB MS spectra were recorded on a JEOL JMX-DM-303, or a JEOL JMX-AX500 using 3-nitrobenzyl alcohol matrix. MALDI TOF-MS spectra were recorded on a Perseptive Biosystems Voyager DE using $\alpha$-cyano-4-hydroxycinnamic acid as a matrix. CD spectra were measured on a JASCO J-720 spectropolarimeter. Vapor pressure osmometry (VPO) was conducted with a KNAUER K-7000 molecular weight apparatus using benzil as a standard. Gel permeation chromatography (GPC) was conducted with a Recycling Preparative HPLC LC-908 or a LC-918 (Japan Analytical Industry, Co. Ltd.).

Dimer 2. Under an argon atmosphere, a mixture of $\mathbf{1 7}^{14}(140 \mathrm{mg}, \quad 0.25$ mmol$)$, tris(dibenzylideneacetone)dipalladium(0) chloroform adduct (13 mg, $0.013 \mathrm{mmol}$ ), cuprous iodide (29 $\mathrm{mg}, 0.15$ $\mathrm{mmol})$, trimesitylphosphine $(15 \mathrm{mg}, 0.039 \mathrm{mmol})$, triphenylphosphine (10 $\mathrm{mg}, 0.038 \mathrm{mmol})$, tetrabutylammonium iodide $(370 \mathrm{mg}, 1.00 \mathrm{mmol})$, triethylamine $(0.4 \mathrm{~mL})$, and $N, N$-dimethylformamide $(8 \mathrm{~mL})$ was freeze-evacuated three times in a flask A. In a flask B, a solution of $(P)-\mathbf{1 6}^{14}(190 \mathrm{mg}, 0.51 \mathrm{mmol})$ in $N, N$-dimethylformamide $(2 \mathrm{~mL})$ was freeze-evacurated three times, and was added dropwise to the flask A. The mixture was stirred for $1 \mathrm{~h}$ at room temperature. The reaction was quenched by adding saturated aqueous ammonium chloride, and the organic materials were extracted with toluene. The organic layer was washed with brine, and dried over magnesium sulfate. The solvent was evaporated under reduced pressure, and separation by recycling GPC gave 2 (239 mg, $0.24 \mathrm{mmol}$, 94\%). Mp 112-114 ${ }^{\circ} \mathrm{C}$ (toluene-methanol). $[\alpha]_{\mathrm{D}}{ }^{22}-567$ (c 1.03, $\left.\mathrm{CHCl}_{3}\right)$. MS (FAB, NBA) $m / z 1010\left(\mathrm{M}^{+}\right)$. UV-vis $\left(\mathrm{CHCl}_{3}, 5 \mu \mathrm{M}\right) \lambda_{\max }(\varepsilon) 335 \mathrm{~nm}\left(1.6 \times 10^{5}\right) . \mathrm{CD}\left(\mathrm{CHCl}_{3}, 5 \mu \mathrm{M}\right) \lambda(\Delta \varepsilon) 298 \mathrm{~nm}(-41), 331 \mathrm{~nm}(55), 383 \mathrm{~nm}(-93)$. IR (KBr) 2207, 2146, $1723 \mathrm{~cm}^{-1}$. Anal. $\left(\mathrm{C}_{71} \mathrm{H}_{70} \mathrm{O}_{2} \mathrm{Si}_{2}\right)$ Calcd for: $\mathrm{C}, 84.31 ; \mathrm{H}, 6.98 \%$. Found: $\mathrm{C}, 84.06$; $\mathrm{H}, 6.96 \%$. ${ }^{1} \mathrm{H}$ NMR $\left(600 \mathrm{MHz}, \mathrm{CDCl}_{3}\right) \delta 0.39(18 \mathrm{H}, \mathrm{s}), 0.86(3 \mathrm{H}, \mathrm{t}, J=7.1 \mathrm{~Hz}), 1.22-1.43(12 \mathrm{H}, \mathrm{m}), 1.50(2 \mathrm{H}$, quin, $J=7.6$ $\mathrm{Hz}), 1.85(2 \mathrm{H}$, quin, $J=7.2 \mathrm{~Hz}), 1.94(6 \mathrm{H}, \mathrm{s}), 1.95(6 \mathrm{H}, \mathrm{s}), 4.42(2 \mathrm{H}, \mathrm{t}, J=6.7 \mathrm{~Hz}), 7.45(2 \mathrm{H}, \mathrm{d}, J=7.2 \mathrm{~Hz}), 7.47$ $(2 \mathrm{H}, \mathrm{d}, J=7.2 \mathrm{~Hz}), 7.66(2 \mathrm{H}, \mathrm{dd}, J=7.2,7.9 \mathrm{~Hz}), 7.71(2 \mathrm{H}, \mathrm{dd}, J=7.2,7.9 \mathrm{~Hz}), 8.03(2 \mathrm{H}, \mathrm{s}), 8.07(2 \mathrm{H}, \mathrm{s}), 8.18$ $(1 \mathrm{H}, \mathrm{t}, J=1.6 \mathrm{~Hz}), 8.35(2 \mathrm{H}, \mathrm{d}, J=1.6 \mathrm{~Hz}), 8.43(2 \mathrm{H}, \mathrm{d}, J=7.9 \mathrm{~Hz}), 8.53(2 \mathrm{H}, \mathrm{d}, J=7.9 \mathrm{~Hz}) .{ }^{13} \mathrm{C} \mathrm{NMR}(150 \mathrm{MHz}$, $\left.\mathrm{CDCl}_{3}\right) \delta 0.13,14.1,22.7,23.1,23.2,26.1,28.7,29.3,29.5,29.6,31.9,65.8,89.3,92.9,100.2,103.1,119.7,120.3$, $123.6,123.7,124.3,126.7,126.9,126.9,129.1,129.2,129.9,129.9,130.9,131.0,131.5,132.1,132.4,132.4,136.8$, $136.9,138.3,165.5$.

Deprotected dimer 10. To a solution of $2(239 \mathrm{mg}, 0.24 \mathrm{mmol})$ in tetrahydrofuran $(3.9 \mathrm{~mL})$ was added $1.0 \mathrm{M}$ tetrabutylammonium fluoride in tetrahydrofuran $(0.71 \mathrm{~mL}, 0.71 \mathrm{mmol})$ at $0{ }^{\circ} \mathrm{C}$. After being stirred for $10 \mathrm{~min}$ at the temperature, saturated aqueous ammonium chloride was added. The organic materials were extracted with ethyl 
acetate. The organic layer was washed with brine, and dried over magnesium sulfate. The solvents were evaporated under reduced pressure, and silica gel chromatography gave $10(205 \mathrm{mg}, 0.24 \mathrm{mmol}, 100 \%) . \mathrm{Mp} 134-136{ }^{\circ} \mathrm{C}$ (toluene-methanol). $[\alpha]_{\mathrm{D}}{ }^{22}-548\left(c 1.02, \mathrm{CHCl}_{3}\right)$. MS (FAB, NBA) $m / z 866\left(\mathrm{M}^{+}\right)$. UV-vis $\left(\mathrm{CHCl}_{3}, 5 \mu \mathrm{M}\right) \lambda_{\max }(\varepsilon)$ $331 \mathrm{~nm}\left(1.4 \times 10^{5}\right) . \mathrm{CD}\left(\mathrm{CHCl}_{3}, 5 \mu \mathrm{M}\right) \lambda(\Delta \varepsilon) 296 \mathrm{~nm}(-37), 330 \mathrm{~nm}(56), 382 \mathrm{~nm}(-79)$. IR (KBr) 3291, 2207, 2101, $1722 \mathrm{~cm}^{-1}$. Anal. $\left(\mathrm{C}_{65} \mathrm{H}_{54} \mathrm{O}_{2}\right)$ Calcd for: $\mathrm{C}, 90.03$; H, 6.28\%. Found: C, 89.74; H, 6.38\%. ${ }^{1} \mathrm{H}$ NMR (600 MHz, $\left.\mathrm{CDCl}_{3}\right) \delta 0.86(3 \mathrm{H}, \mathrm{t}, J=6.9 \mathrm{~Hz}), 1.22-1.43(12 \mathrm{H}, \mathrm{m}), 1.50(2 \mathrm{H}$, quin, $J=6.9 \mathrm{~Hz}), 1.85(2 \mathrm{H}$, quin, $J=6.9 \mathrm{~Hz})$, $1.96(12 \mathrm{H}, \mathrm{s}), 3.57(2 \mathrm{H}, \mathrm{s}), 4.42(2 \mathrm{H}, \mathrm{t}, J=6.9 \mathrm{~Hz}), 7.47(2 \mathrm{H}, \mathrm{d}, J=7.2 \mathrm{~Hz}), 7.49(2 \mathrm{H}, \mathrm{d}, J=6.9 \mathrm{~Hz}), 7.67(2 \mathrm{H}, \mathrm{dd}$, $J=7.2,8.2 \mathrm{~Hz}), 7.73(2 \mathrm{H}, \mathrm{dd}, J=6.9,8.3 \mathrm{~Hz}), 8.07(2 \mathrm{H}, \mathrm{s}), 8.10(2 \mathrm{H}, \mathrm{s}), 8.18(1 \mathrm{H}, \mathrm{t}, J=1.7 \mathrm{~Hz}), 8.35(2 \mathrm{H}, \mathrm{d}, J=$ $1.7 \mathrm{~Hz}), 8.45(2 \mathrm{H}, \mathrm{d}, J=8.2 \mathrm{~Hz}), 8.54(2 \mathrm{H}, \mathrm{d}, J=8.3 \mathrm{~Hz}) .{ }^{13} \mathrm{C} \mathrm{NMR}\left(150 \mathrm{MHz}, \mathrm{CDCl}_{3}\right) \delta 14.1,22.7,23.2,26.1$, 28.7, 29.3, 29.5, 29.6, 31.9, 65.8, 81.8, 82.4, 89.3, 92.9, 119.4, 119.8, 123.6, 123.6, 124.3, 126.8, 127.0, 127.0, $129.2,129.3,129.8,130.4,130.9,131.0,131.5,132.2,132.4,136.9,136.9,138.3,165.5$.

Tetramer 4 (Typical procedure for the Sonogashira coupling reaction). Under an argon atmosphere, a mixture of $(P)-\mathbf{1}^{14}$ (114 mg, $\left.0.14 \mathrm{mmol}\right)$, tris(dibenzylideneacetone)dipalladium(0) chloroform adduct (3.7 $\left.\mathrm{mg}, 3.6 \mu \mathrm{mol}\right)$, cuprous iodide $(8.3 \mathrm{mg}, 0.044 \mathrm{mmol})$, trimesitylphosphine $(8.4 \mathrm{mg}, 0.022 \mathrm{mmol})$, triphenylphosphine $(5.7 \mathrm{mg}$, $0.022 \mathrm{mmol})$, tetrabutylammonium iodide $(107 \mathrm{mg}, 0.29 \mathrm{mmol})$, triethylamine $(0.090 \mathrm{~mL})$, and $\mathrm{N}, \mathrm{N}$-dimethylformamide $(1.7 \mathrm{~mL})$ was freeze-evacuated three times in a flask A. In a flask $\mathrm{B}$, a solution of $\mathbf{1 0}$ (63 $\mathrm{mg}, 0.072 \mathrm{mmol})$ in toluene $(2.2 \mathrm{~mL})$ was freeze-evacuated three times, and was added dropwise to the flask A. The mixture was stirred for $2 \mathrm{~h}$ at $45{ }^{\circ} \mathrm{C}$. The reaction was quenched by adding saturated aqueous ammonium chloride, and the organic materials were extracted with toluene. The organic layer was washed with brine, and dried over magnesium sulfate. The solvents were evaporated under reduced pressure, and separation by recycling GPC gave 4 (135 mg, $0.063 \mathrm{mmol}, 87 \%)$. Mp 148-149 ${ }^{\circ} \mathrm{C}$ (toluene-methanol). $[\alpha]_{\mathrm{D}}{ }^{23}-546$ (c 1.02, $\mathrm{CHCl}_{3}$ ). MALDI TOF-MS $m / z$ Calcd for ${ }^{12} \mathrm{C}_{152}{ }^{13} \mathrm{CH}_{146} \mathrm{O}_{6} \mathrm{Si}_{2}$ : 2136.1. Found: 2136.9. UV-vis $\left(\mathrm{CHCl}_{3}, 5 \mu \mathrm{M}\right) \lambda_{\max }(\varepsilon) 337 \mathrm{~nm}$ $\left(2.9 \times 10^{5}\right) . \mathrm{CD}\left(\mathrm{CHCl}_{3}, 5 \mu \mathrm{M}\right) \lambda(\Delta \varepsilon) 296 \mathrm{~nm}(-78), 332 \mathrm{~nm}(77), 388 \mathrm{~nm}(-190)$. IR (KBr) 2208, 2146, $1721 \mathrm{~cm}^{-1}$. Anal. $\left(\mathrm{C}_{153} \mathrm{H}_{146} \mathrm{O}_{6} \mathrm{Si}_{2}\right)$ Calcd for: $\mathrm{C}, 85.99 ; \mathrm{H}, 6.89 \%$. Found: C, 85.94; H, 6.96\%. ${ }^{1} \mathrm{H}$ NMR $\left(600 \mathrm{MHz}, \mathrm{CDCl}_{3}\right) \delta$ $0.39(18 \mathrm{H}, \mathrm{s}), 0.86(9 \mathrm{H}, \mathrm{t}, J=6.9 \mathrm{~Hz}), 1.20-1.43(36 \mathrm{H}, \mathrm{m}), 1.45-1.54(6 \mathrm{H}, \mathrm{m}), 1.85(6 \mathrm{H}$, quin, $J=7.2 \mathrm{~Hz}), 1.93$ $(6 \mathrm{H}, \mathrm{s}), 1.95(6 \mathrm{H}, \mathrm{s}), 1.99(12 \mathrm{H}, \mathrm{s}), 4.40-4.43(6 \mathrm{H}, \mathrm{m}), 7.45(2 \mathrm{H}, \mathrm{d}, J=7.1 \mathrm{~Hz}), 7.47(2 \mathrm{H}, \mathrm{d}, J=7.6 \mathrm{~Hz}), 7.51(4 \mathrm{H}$, $\mathrm{d}, J=6.9 \mathrm{~Hz}), 7.66(2 \mathrm{H}, \mathrm{dd}, J=7.1,8.1 \mathrm{~Hz}), 7.70-7.75(6 \mathrm{H}, \mathrm{m}), 8.01(2 \mathrm{H}, \mathrm{s}), 8.06(2 \mathrm{H}, \mathrm{s}), 8.10(4 \mathrm{H}, \mathrm{s}), 8.19(2 \mathrm{H}$, $\mathrm{t}, J=1.4 \mathrm{~Hz}), 8.20(1 \mathrm{H}, \mathrm{t}, J=1.5 \mathrm{~Hz}), 8.35(2 \mathrm{H}, \mathrm{d}, J=1.5 \mathrm{~Hz}), 8.36-8.37(4 \mathrm{H}, \mathrm{m}), 8.43(2 \mathrm{H}, \mathrm{d}, J=8.1 \mathrm{~Hz})$, 8.53-8.57 (6H, m). ${ }^{13} \mathrm{C}$ NMR $\left(150 \mathrm{MHz}, \mathrm{CDCl}_{3}\right) \delta 0.12,14.1,22.7,23.1,23.2,23.2,26.0,28.7,29.3,29.6,31.9$, $65.8,89.3,92.9,93.0,100.2,103.1,119.7,119.9,120.3,123.6,123.6,124.3,126.7,126.9,127.0,129.1,129.2$, $129.3,129.9,130.9,131.0,131.1,131.5,132.1,132.2,132.4,136.8,136.9,136.9,138.3,165.4$.

Deprotected tetramer 12 (Typical procedure for desilylation). To a solution of 4 (171 $\mathrm{mg}, 0.080 \mathrm{mmol})$ in tetrahydrofuran $(1.3 \mathrm{~mL})$ was added $1.0 \mathrm{M}$ tetrabutylammonium in tetrahydrofuran $(0.24 \mathrm{~mL}, 0.24 \mathrm{mmol})$ at $0{ }^{\circ} \mathrm{C}$. After being stirred for $10 \mathrm{~min}$ at the temperature, saturated aqueous ammonium chloride was added. The organic materials were extracted with ethyl acetate. The organic layer was washed with brine, and dried over magnesium sulfate. The solvents were evaporated under reduced pressure, and silica gel chromatography gave 12 (159 mg, $0.080 \mathrm{mmol}, 100 \%)$. Mp $148-152^{\circ} \mathrm{C}$ (toluene-methanol). $[\alpha]_{\mathrm{D}}{ }^{23}-564$ (c 1.02, $\mathrm{CHCl}_{3}$ ). MALDI TOF-MS $m / z$ Calcd for ${ }^{12} \mathrm{C}_{146}{ }^{13} \mathrm{CH}_{130} \mathrm{O}_{6}$ : 1992.0. Found: 1992.3. UV-vis $\left(\mathrm{CHCl}_{3}, 5 \mu \mathrm{M}\right) \lambda_{\max }(\varepsilon) 332 \mathrm{~nm}\left(2.6 \times 10^{5}\right)$. CD $\left(\mathrm{CHCl}_{3}, 5\right.$ $\mu \mathrm{M}) \lambda(\Delta \varepsilon) 296 \mathrm{~nm}(-71), 331 \mathrm{~nm}(77), 387 \mathrm{~nm}(-176)$. IR (KBr) 3302, 2207, 2101, $1720 \mathrm{~cm}^{-1}$. Anal. $\left(\mathrm{C}_{147} \mathrm{H}_{130} \mathrm{O}_{6}\right)$ Calcd for: C, 88.61; H, 6.58\%. Found: C, 88.33; H, 6.55\%. ${ }^{1} \mathrm{H}$ NMR $\left(600 \mathrm{MHz}, \mathrm{CDCl}_{3}\right) \delta 0.85(9 \mathrm{H}, \mathrm{t}, J=7.1 \mathrm{~Hz})$, $1.20-1.44$ (36H, m), 1.46-1.53 (6H, m), 1.82-1.88 (6H, m), $1.94(6 \mathrm{H}, \mathrm{s}), 1.94(6 \mathrm{H}, \mathrm{s}), 1.99(12 \mathrm{H}, \mathrm{s}), 3.55(2 \mathrm{H}, \mathrm{s})$, 
4.40-4.44 (6H, m), $7.45(2 \mathrm{H}, \mathrm{d}, J=6.9 \mathrm{~Hz}), 7.47(2 \mathrm{H}, \mathrm{d}, J=6.9 \mathrm{~Hz}), 7.50(4 \mathrm{H}, \mathrm{d}, J=6.9 \mathrm{~Hz}), 7.65(2 \mathrm{H}, \mathrm{dd}, J=$ 6.9, $7.9 \mathrm{~Hz}), 7.70-7.74(6 \mathrm{H}, \mathrm{m}), 8.03(2 \mathrm{H}, \mathrm{s}), 8.06(2 \mathrm{H}, \mathrm{s}), 8.09(4 \mathrm{H}, \mathrm{s}), 8.17(2 \mathrm{H}, \mathrm{t}, J=1.6 \mathrm{~Hz}), 8.19(1 \mathrm{H}, \mathrm{t}, J=$ $1.4 \mathrm{~Hz}), 8.34(2 \mathrm{H}, \mathrm{d}, J=1.4 \mathrm{~Hz}), 8.35-8.36(4 \mathrm{H}, \mathrm{m}), 8.43(2 \mathrm{H}, \mathrm{d}, J=7.9 \mathrm{~Hz}), 8.53-8.56(6 \mathrm{H}, \mathrm{m}) .{ }^{13} \mathrm{C}$ NMR $(150$ $\left.\mathrm{MHz}, \mathrm{CDCl}_{3}\right) \delta 14.1,22.7,23.2,23.2,26.0,28.7,29.3,29.3,29.6,31.9,65.8,82.4,89.3,89.3,93.0,93.0,119.4$, $119.8,119.8,123.5,123.6,123.6,124.3,126.8,126.8,126.9,127.0,127.0,129.2,129.2,129.3,129.8,129.9,130.4$, $130.8,130.9,131.0,131.1,131.5,132.2,132.2,132.4,136.8,136.9,136.9,138.3,165.4$.

Pentamer 5. The compound (152 mg, $0.056 \mathrm{mmol}, 99 \%)$ was prepared from $\mathbf{1 1}^{14}(82 \mathrm{mg}, 0.057 \mathrm{mmol})$ and $(P)-\mathbf{1}$ (90 mg, $0.11 \mathrm{mmol})$. Mp 138-140 ${ }^{\circ} \mathrm{C}$ (toluene-methanol). $[\alpha]_{\mathrm{D}}{ }^{22}-551$ (c 1.02, $\mathrm{CHCl}_{3}$ ). MALDI TOF-MS $m / z$ Calcd for ${ }^{12} \mathrm{C}_{193}{ }^{13} \mathrm{CH}_{184} \mathrm{O}_{8} \mathrm{Si}_{2}$ : 2698.4. Found: 2699.1. UV-vis $\left(\mathrm{CHCl}_{3}, 5 \mu \mathrm{M}\right) \lambda_{\max }(\varepsilon) 337 \mathrm{~nm}\left(3.6 \times 10^{5}\right)$. CD $\left(\mathrm{CHCl}_{3}, 5\right.$ $\mu \mathrm{M}) \lambda(\Delta \varepsilon) 297 \mathrm{~nm}(-92), 334 \mathrm{~nm}(88), 389 \mathrm{~nm}(-239)$. IR (KBr) 2206, 2146, $1722 \mathrm{~cm}^{-1}$. Anal. $\left(\mathrm{C}_{194} \mathrm{H}_{184} \mathrm{O}_{8} \mathrm{Si}_{2}\right)$ Calcd for: C, 86.31; H, 6.87\%. Found: C, 86.36; H, 6.96\%. ${ }^{1} \mathrm{H}$ NMR $\left(600 \mathrm{MHz}, \mathrm{CDCl}_{3}\right) \delta 0.38(18 \mathrm{H}, \mathrm{s}), 0.85(12 \mathrm{H}$, $\mathrm{t}, J=7.2 \mathrm{~Hz}), 1.20-1.43(48 \mathrm{H}, \mathrm{m}), 1.46-1.52(8 \mathrm{H}, \mathrm{m}), 1.81-1.88(8 \mathrm{H}, \mathrm{m}), 1.93(6 \mathrm{H}, \mathrm{s}), 1.94(6 \mathrm{H}, \mathrm{s}), 1.99(18 \mathrm{H}, \mathrm{s})$, 4.40-4.43 (8H, m), $7.44(2 \mathrm{H}, \mathrm{d}, J=7.2 \mathrm{~Hz}), 7.47(2 \mathrm{H}, \mathrm{d}, J=7.6 \mathrm{~Hz}), 7.50(6 \mathrm{H}, \mathrm{d}, J=6.9 \mathrm{~Hz}), 7.66(2 \mathrm{H}, \mathrm{dd}, J=$ $7.2,8.1 \mathrm{~Hz}), 7.70-7.75(8 \mathrm{H}, \mathrm{m}), 8.01(2 \mathrm{H}, \mathrm{s}), 8.06(2 \mathrm{H}, \mathrm{s}), 8.12(6 \mathrm{H}, \mathrm{s}), 8.19(2 \mathrm{H}, \mathrm{t}, J=1.6 \mathrm{~Hz}), 8.20(2 \mathrm{H}, \mathrm{t}, J=$ $1.6 \mathrm{~Hz}), 8.35(2 \mathrm{H}, \mathrm{d}, J=1.6 \mathrm{~Hz}), 8.36-8.37(6 \mathrm{H}, \mathrm{m}), 8.42(2 \mathrm{H}, \mathrm{d}, J=8.1 \mathrm{~Hz}), 8.53-8.57(8 \mathrm{H}, \mathrm{m}) .{ }^{13} \mathrm{C}$ NMR $(150$ $\left.\mathrm{MHz}, \mathrm{CDCl}_{3}\right) \delta 0.11,14.1,22.7,23.1,23.2,23.2,26.0,28.7,29.3,29.5,31.9,65.8,89.3,89.3,89.4,92.9,93.0$, $100.2,103.1,119.7,119.9,120.3,123.6,123.6,123.7,124.3,124.3,126.7,126.9,126.9,126.9,127.0,129.1,129.2$, $129.3,129.9,130.9,131.0,131.0,131.1,131.5,131.5,132.1,132.2,132.4,132.4,136.8,136.9,137.0,138.3$, 165.4 .

Deprotected pentamer 13. The compound (118 mg, $0.046 \mathrm{mmol}, 99 \%)$ was prepared from 5 (127 $\mathrm{mg}, 0.047$ mmol). Mp $134-136{ }^{\circ} \mathrm{C}$ (toluene-methanol). $[\alpha]_{\mathrm{D}}^{22}-540$ (c 1.01, $\mathrm{CHCl}_{3}$ ). MALDI TOF-MS $\mathrm{m} / z$ Calcd for ${ }^{12} \mathrm{C}_{187}{ }^{13} \mathrm{CH}_{168} \mathrm{O}_{8}$ : 2554.3. Found: 2555.0. UV-vis $\left(\mathrm{CHCl}_{3}, 5 \mu \mathrm{M}\right) \lambda_{\max }(\varepsilon) 333 \mathrm{~nm}\left(3.3 \times 10^{5}\right)$. CD $\left(\mathrm{CHCl}_{3}, 5 \mu \mathrm{M}\right) \lambda$ $(\Delta \varepsilon) 297 \mathrm{~nm}(-82), 331 \mathrm{~nm}(90), 388 \mathrm{~nm}(-223)$. IR (KBr) 3305, 2206, 2103, $1720 \mathrm{~cm}^{-1}$. Anal. $\left(\mathrm{C}_{188} \mathrm{H}_{168} \mathrm{O}_{8}\right)$ Calcd for: C, 88.36; H, 6.63\%. Found: C, 88.11; H, 6.73\%. ${ }^{1} \mathrm{H}$ NMR $\left(600 \mathrm{MHz}, \mathrm{CDCl}_{3}\right) \delta 0.85(12 \mathrm{H}, \mathrm{t}, J=6.9 \mathrm{~Hz})$, $1.20-1.43$ (48H, m), 1.46-1.53 (8H, m), 1.82-1.88 (8H, m), $1.94(6 \mathrm{H}, \mathrm{s}), 1.94$ (6H, s), $1.98(12 \mathrm{H}, \mathrm{s}), 1.99$ (6H, s), $3.56(2 \mathrm{H}, \mathrm{s}), 4.40-4.43(8 \mathrm{H}, \mathrm{m}), 7.45(2 \mathrm{H}, \mathrm{d}, J=6.9 \mathrm{~Hz}), 7.48(2 \mathrm{H}, \mathrm{d}, J=6.9 \mathrm{~Hz}), 7.49-7.51(6 \mathrm{H}, \mathrm{m}), 7.65(2 \mathrm{H}, \mathrm{dd}$, $J=6.9,7.9 \mathrm{~Hz}), 7.71-7.75(8 \mathrm{H}, \mathrm{m}), 8.04(2 \mathrm{H}, \mathrm{s}), 8.07(2 \mathrm{H}, \mathrm{s}), 8.12(4 \mathrm{H}, \mathrm{s}), 8.13(2 \mathrm{H}, \mathrm{s}), 8.18(2 \mathrm{H}, \mathrm{t}, J=1.4 \mathrm{~Hz})$, $8.20(2 \mathrm{H}, \mathrm{t}, J=1.4 \mathrm{~Hz}), 8.34(2 \mathrm{H}, \mathrm{d}, J=1.4 \mathrm{~Hz}), 8.35(2 \mathrm{H}, \mathrm{d}, J=1.4 \mathrm{~Hz}), 8.36-8.37(4 \mathrm{H}, \mathrm{m}), 8.44(2 \mathrm{H}, \mathrm{d}, J=7.9$ $\mathrm{Hz}), 8.53-8.57(8 \mathrm{H}, \mathrm{m}) .{ }^{13} \mathrm{C} \mathrm{NMR}\left(150 \mathrm{MHz}, \mathrm{CDCl}_{3}\right) \delta 14.1,22.7,23.2,23.2,26.1,28.8,29.3,29.3,29.6,31.9$, $65.8,81.8,82.4,89.3,89.3,93.0,93.0,119.4,119.8,119.9,119.9,123.6,123.6,123.6,124.3,126.8,126.9,126.9$, $127.0,127.0,129.2,129.2,129.3,129.8,129.9,130.4,130.8,130.9,131.0,131.0,131.1,131.5,131.5,132.2,132.2$, $132.2,132.4,132.4,136.8,136.9,136.9,137.0,138.3,138.3,165.4$.

Hexamer 6. The compound (163 mg, $0.050 \mathrm{mmol}, 83 \%)$ was prepared from 12 (122 mg, $0.061 \mathrm{mmol})$ and $(P)-\mathbf{1}$ (94 mg, $0.12 \mathrm{mmol}$ ). Mp $144-148{ }^{\circ} \mathrm{C}$ (toluene-methanol). $[\alpha]_{\mathrm{D}}{ }^{23}-536$ (c $0.10, \mathrm{CHCl}_{3}$ ). MALDI TOF-MS $\mathrm{m} / \mathrm{z}$ Calcd for ${ }^{12} \mathrm{C}_{233}{ }^{13} \mathrm{C}_{2} \mathrm{H}_{222} \mathrm{O}_{10} \mathrm{Si}_{2}$ : 3261.6. Found: 3261.1. UV-vis $\left(\mathrm{CHCl}_{3}, 1 \mu \mathrm{M}\right) \lambda_{\max }(\varepsilon) 337 \mathrm{~nm}\left(4.2 \times 10^{5}\right)$. CD $\left(\mathrm{CHCl}_{3}, 5 \mu \mathrm{M}\right) \lambda(\Delta \varepsilon) 297 \mathrm{~nm}(-103), 333 \mathrm{~nm}(105), 389 \mathrm{~nm}(-283)$. IR (KBr) 2209, 2147, $1722 \mathrm{~cm}^{-1}$. Anal. $\left(\mathrm{C}_{235} \mathrm{H}_{222} \mathrm{O}_{10} \mathrm{Si}_{2}\right)$ Calcd for: C, 86.52; H, 6.86\%. Found: C, 86.41; H, 6.92\%. ${ }^{1} \mathrm{H}$ NMR $\left(600 \mathrm{MHz}, \mathrm{CDCl}_{3}\right) \delta 0.38$ $(18 \mathrm{H}, \mathrm{s}), 0.85(15 \mathrm{H}, \mathrm{t}, J=6.9 \mathrm{~Hz}), 1.20-1.44(60 \mathrm{H}, \mathrm{m}), 1.47-1.55(10 \mathrm{H}, \mathrm{m}), 1.81-1.89(10 \mathrm{H}, \mathrm{m}), 1.93(6 \mathrm{H}, \mathrm{s})$, $1.94(6 \mathrm{H}, \mathrm{s}), 1.98(12 \mathrm{H}, \mathrm{s}), 1.99(12 \mathrm{H}, \mathrm{s}), 4.40-4.43(10 \mathrm{H}, \mathrm{m}), 7.44(2 \mathrm{H}, \mathrm{d}, J=7.2 \mathrm{~Hz}), 7.47(2 \mathrm{H}, \mathrm{d}, J=7.3 \mathrm{~Hz})$, 7.48-7.54 (8H, m), $7.66(2 \mathrm{H}, \mathrm{dd}, J=7.2,7.9 \mathrm{~Hz}), 7.70-7.76(10 \mathrm{H}, \mathrm{m}), 8.02(2 \mathrm{H}, \mathrm{s}), 8.07(2 \mathrm{H}, \mathrm{s}), 8.14(4 \mathrm{H}, \mathrm{s}), 8.16$ (4H, s), 8.18-8.19 (2H, m), 8.19-8.22 (3H, m), 8.34-8.35 (2H, m), 8.35-8.36 (4H, m), 8.36-8.38 (4H, m), 8.42 
$(2 \mathrm{H}, \mathrm{d}, J=7.9 \mathrm{~Hz}), 8.53-8.57(10 \mathrm{H}, \mathrm{m}) .{ }^{13} \mathrm{C} \mathrm{NMR}\left(150 \mathrm{MHz}, \mathrm{CDCl}_{3}\right) \delta 0.11,14.1,22.7,23.1,23.2,23.2,26.1$, 28.8, 29.3, 29.5, 31.9, 65.8, 89.3, 89.4, 92.9, 93.0, 100.2, 103.1, 119.7, 119.9, 120.3, 123.6, 123.6, 123.6, 123.7, $124.3,124.3,126.7,126.9,126.9,127.0,129.1,129.2,129.3,129.9,130.9,131.0,131.0,131.1,131.5,132.1,132.2$, $132.4,136.8,136.9,137.0,138.3,165.5$.

Deprotected hexamer 14. The compound (145 mg, $0.047 \mathrm{mmol}, 95 \%)$ was prepared from 6 (161 mg, $0.049 \mathrm{mmol})$. $\mathrm{Mp} \quad{ }^{145-147}{ }^{\circ} \mathrm{C}$ (toluene-methanol). $[\alpha]_{\mathrm{D}}{ }^{23}-466$ (c $0.10, \mathrm{CHCl}_{3}$ ). MALDI TOF-MS $\mathrm{m} / \mathrm{z}$ Calcd for: ${ }^{12} \mathrm{C}_{227}{ }^{13} \mathrm{C}_{2} \mathrm{H}_{206} \mathrm{O}_{10}$ 3117.6. Found: 3118.1. UV-vis $\left(\mathrm{CHCl}_{3}, 1 \mu \mathrm{M}\right) \lambda_{\max }(\varepsilon) 334 \mathrm{~nm}\left(3.8 \times 10^{5}\right)$. CD $\left(\mathrm{CHCl}_{3}, 5 \mu \mathrm{M}\right) \lambda$ $(\Delta \varepsilon) 298 \mathrm{~nm}$ (-99), $332 \mathrm{~nm}$ (105), $389 \mathrm{~nm}(-265)$. IR (KBr) 3307, 2205, 2101, $1720 \mathrm{~cm}^{-1}$. Anal. $\left(\mathrm{C}_{229} \mathrm{H}_{206} \mathrm{O}_{10}\right)$ Calcd for: $\mathrm{C}, 88.21 ; \mathrm{H}, 6.66 \%$. Found: $\mathrm{C}, 87.81 ; \mathrm{H}, 6.89 \% .{ }^{1} \mathrm{H} \mathrm{NMR}\left(600 \mathrm{MHz}, \mathrm{CDCl}_{3}\right) \delta 0.85(15 \mathrm{H}, \mathrm{t}, J=7.1 \mathrm{~Hz})$, 1.20-1.43 (60H, m), 1.46-1.53 (10H, m), 1.81-1.89 (10H, m), $1.94(6 \mathrm{H}, \mathrm{s}), 1.94(6 \mathrm{H}, \mathrm{s}), 1.97(12 \mathrm{H}, \mathrm{s}), 1.98(6 \mathrm{H}, \mathrm{s})$, $1.99(6 \mathrm{H}, \mathrm{s}), 3.56(2 \mathrm{H}, \mathrm{s}), 4.40-4.43(10 \mathrm{H}, \mathrm{m}), 7.45(2 \mathrm{H}, \mathrm{d}, J=6.9 \mathrm{~Hz}), 7.47-7.51(10 \mathrm{H}, \mathrm{m}), 7.66(2 \mathrm{H}, \mathrm{dd}, J=6.9$, $7.6 \mathrm{~Hz}), 7.71-7.75(10 \mathrm{H}, \mathrm{m}), 8.06(2 \mathrm{H}, \mathrm{s}), 8.09(2 \mathrm{H}, \mathrm{s}), 8.14(4 \mathrm{H}, \mathrm{s}), 8.15(4 \mathrm{H}, \mathrm{s}), 8.18(2 \mathrm{H}, \mathrm{t}, J=1.6 \mathrm{~Hz}), 8.21$ $(2 \mathrm{H}, \mathrm{t}, J=1.6 \mathrm{~Hz}), 8.22(1 \mathrm{H}, \mathrm{t}, J=1.6 \mathrm{~Hz}), 8.33(2 \mathrm{H}, \mathrm{d}, J=1.6 \mathrm{~Hz}), 8.35-8.36(4 \mathrm{H}, \mathrm{m}), 8.36-8.37(4 \mathrm{H}, \mathrm{m}), 8.44$ $(2 \mathrm{H}, \mathrm{d}, J=7.6 \mathrm{~Hz}), 8.53-8.56(10 \mathrm{H}, \mathrm{m}) .{ }^{13} \mathrm{C} \mathrm{NMR}\left(150 \mathrm{MHz}, \mathrm{CDCl}_{3}\right) \delta$ 14.1, 22.8, 23.2, 23.2, 26.1, 28.6, 29.3, 29.3, 29.6, 31.9, 65.8, 81.8, 82.4, 89.3, 89.3, 93.0, 93.0, 119.4, 119.8, 119.9, 123.5, 123.6, 123.6, 124.3, 126.8, $126.9,126.9,127.0,127.0,129.2,129.2,129.3,129.8,129.9,130.4,130.8,130.9,131.0,131.1,131.5,132.2,132.2$, $132.4,136.8,137.0,138.3,138.3,165.5$.

Heptamer 7. The compound (106 mg, $0.028 \mathrm{mmol}, 87 \%)$ was prepared from 13 (83 $\mathrm{mg}, 0.032 \mathrm{mmol})$ and $(P)-\mathbf{1}$ (51 mg, 0.065 mmol). Mp $150-153{ }^{\circ} \mathrm{C}$ (toluene-methanol). $[\alpha]_{\mathrm{D}}{ }^{24}+4396$ ( $c 0.10$, trifluoromethylbenzene, observed within 30 min after dissolution). MALDI TOF-MS $m / z$ Calcd for ${ }^{12} \mathrm{C}_{274}{ }^{13} \mathrm{C}_{2} \mathrm{H}_{260} \mathrm{O}_{12} \mathrm{Si}_{2}$ : 3823.9. Found: 3823.5 . UV-vis (trifluoromethylbenzene, $1 \mu \mathrm{M}$, observed within $10 \mathrm{~min}$ after dissolution) $\lambda_{\max }(\varepsilon) 332 \mathrm{~nm}\left(2.9 \times 10^{5}\right)$. CD $\left(\mathrm{CHCl}_{3}, 5 \mu \mathrm{M}\right.$, observed $5 \mathrm{~min}$ after dissolution) $\lambda(\Delta \varepsilon) 329 \mathrm{~nm}(-1170), 366 \mathrm{~nm}(1750), 385 \mathrm{~nm}(1190), 392 \mathrm{~nm}$ (1330). IR (KBr) 2204, 2145, $1724 \mathrm{~cm}^{-1}$. Anal. $\left(\mathrm{C}_{276} \mathrm{H}_{260} \mathrm{O}_{12} \mathrm{Si}_{2}\right)$ Calcd for: C, 86.66; H, 6.85\%. Found: C, 86.37; H, $6.89 \%$. ${ }^{1} \mathrm{H}$ NMR $\left(600 \mathrm{MHz}, \mathrm{CDCl}_{3}, 1 \mathrm{mM}\right.$, observed at $60{ }^{\circ} \mathrm{C}$ after being heated at $60{ }^{\circ} \mathrm{C}$ for $\left.30 \mathrm{~min}\right) \delta 0.38(18 \mathrm{H}$, s), $0.85(18 \mathrm{H}, \mathrm{t}, J=6.8 \mathrm{~Hz}), 1.24-1.45(72 \mathrm{H}, \mathrm{m}), 1.46-1.53(12 \mathrm{H}, \mathrm{m}), 1.82-1.88(12 \mathrm{H}, \mathrm{m}), 1.94(6 \mathrm{H}, \mathrm{s}), 1.95(6 \mathrm{H}$, s), $1.99(30 \mathrm{H}, \mathrm{s}), 4.41-4.43(12 \mathrm{H}, \mathrm{m}), 7.43(2 \mathrm{H}, \mathrm{d}, J=6.9 \mathrm{~Hz}), 7.45(2 \mathrm{H}, \mathrm{d}, J=6.8 \mathrm{~Hz}), 7.48(10 \mathrm{H}, \mathrm{d}, J=6.8 \mathrm{~Hz})$, $7.64(2 \mathrm{H}, \mathrm{dd}, J=6.9,8.3 \mathrm{~Hz}), 7.68-7.73(12 \mathrm{H}, \mathrm{m}), 8.00(2 \mathrm{H}, \mathrm{s}), 8.06(2 \mathrm{H}, \mathrm{s}), 8.12(4 \mathrm{H}, \mathrm{s}), 8.13(6 \mathrm{H}, \mathrm{s}), 8.16-8.17$ $(2 \mathrm{H}, \mathrm{m}), 8.18-8.19(4 \mathrm{H}, \mathrm{m}), 8.33-8.34(2 \mathrm{H}, \mathrm{m}), 8.35-8.36(10 \mathrm{H}, \mathrm{m}), 8.42(2 \mathrm{H}, \mathrm{d}, J=8.3 \mathrm{~Hz}), 8.52(2 \mathrm{H}, \mathrm{d}, J=7.6$ $\mathrm{Hz}), 8.55(10 \mathrm{H}, \mathrm{d}, J=8.2 \mathrm{~Hz}) \cdot{ }^{13} \mathrm{C}$ NMR $\left(150 \mathrm{MHz}, \mathrm{CDCl}_{3}, 1 \mathrm{mM}\right.$, observed at $60{ }^{\circ} \mathrm{C}$ after being heated at $60{ }^{\circ} \mathrm{C}$ for $30 \mathrm{~min}) \delta 0.13,14.0,22.7,23.1,23.1,23.2,26.1,28.8,29.3,29.3,29.6,31.9,65.8,89.5,93.0,93.1,100.3$, $103.3,120.0,123.6,123.7,123.8,124.5,126.9,127.0,127.0,127.1,129.1,129.2,129.3,129.9,131.0,131.1,131.2$, $131.8,132.3,132.4,132.4,132.6,136.9,137.0,137.1,138.3,165.5$.

Deprotected heptamer 15. The compound ( $33 \mathrm{mg}, 9.0 \mu \mathrm{mol}, 96 \%$ ) was prepared from 7 (36 mg, $9.4 \mu \mathrm{mol})$. Mp 198-200 ${ }^{\circ} \mathrm{C}$ (toluene-methanol). $[\alpha]_{\mathrm{D}}{ }^{25}+4610$ (c 0.10, trifluoromethylbenzene, observed within 30 min after dissolutin). MALDI TOF-MS $m / z$ Calcd for ${ }^{12} \mathrm{C}_{268}{ }^{13} \mathrm{C}_{2} \mathrm{H}_{244} \mathrm{O}_{12}$ : 3679.8. Found: 3679.2. UV-vis (trifluoromethylbenzene, $1 \mu \mathrm{M}$, observed within $10 \mathrm{~min}$ after dissolution) $\lambda_{\max }(\varepsilon) 323 \mathrm{~nm}\left(2.7 \times 10^{5}\right)$. IR $(\mathrm{KBr})$ 3306, 2206, 2101, $1722 \mathrm{~cm}^{-1}$. Anal. $\left(\mathrm{C}_{270} \mathrm{H}_{244} \mathrm{O}_{12}\right)$ Calcd for: $\mathrm{C}, 88.10 ; \mathrm{H}, 6.68 \%$. Found: C, 87.97; H, 6.80\%. ${ }^{1} \mathrm{H}$ $\operatorname{NMR}\left(600 \mathrm{MHz}, \mathrm{CDCl}_{3}, 5 \mathrm{mM}\right.$, observed at $60{ }^{\circ} \mathrm{C}$ after being heated at $60{ }^{\circ} \mathrm{C}$ for $\left.1 \mathrm{~h}\right) \delta 0.85(18 \mathrm{H}, \mathrm{t}, J=7.3 \mathrm{~Hz})$, $1.20-1.53(84 \mathrm{H}, \mathrm{m}), 1.81-1.88(12 \mathrm{H}, \mathrm{m}), 1.94(6 \mathrm{H}, \mathrm{s}), 1.95(6 \mathrm{H}, \mathrm{s}), 1.98(30 \mathrm{H}, \mathrm{s}), 3.52(2 \mathrm{H}, \mathrm{s}), 4.40-4.43(12 \mathrm{H}, \mathrm{m})$, $7.43(2 \mathrm{H}, \mathrm{d}, J=7.6 \mathrm{~Hz}), 7.44(2 \mathrm{H}, \mathrm{d}, J=7.6 \mathrm{~Hz}), 7.48(10 \mathrm{H}, \mathrm{d}, J=6.9 \mathrm{~Hz}), 7.63(2 \mathrm{H}, \mathrm{dd}, J=7.6,8.2 \mathrm{~Hz})$, $7.68-7.72(12 \mathrm{H}, \mathrm{m}), 8.03(2 \mathrm{H}, \mathrm{s}), 8.06(2 \mathrm{H}, \mathrm{s}), 8.11(4 \mathrm{H}, \mathrm{s}), 8.12(6 \mathrm{H}, \mathrm{s}), 8.16(2 \mathrm{H}, \mathrm{t}, J=1.4 \mathrm{~Hz}), 8.18(4 \mathrm{H}, \mathrm{s})$, 
8.32-8.33 (2H, m), 8.34-8.35 (10H, m), $8.42(2 \mathrm{H}, \mathrm{d}, J=8.2 \mathrm{~Hz}), 8.51-8.54(12 \mathrm{H}, \mathrm{m}) .{ }^{13} \mathrm{C} \mathrm{NMR}\left(150 \mathrm{MHz}, \mathrm{CDCl}_{3}\right.$, $5 \mathrm{mM}$, observed at $60^{\circ} \mathrm{C}$ after being heated at $60^{\circ} \mathrm{C}$ for $\left.1 \mathrm{~h}\right) \delta 14.0,22.6,23.1,23.1,26.1,28.9,29.3,29.3,29.6$, $31.9,65.8,82.0,82.3,89.5,93.1,93.1,119.6,120.0,120.1,123.7,123.7,123.7,124.5,127.0,127.0,127.0,127.1$, $129.2,129.3,129.3,129.9,130.0,130.5,131.0,131.1,131.2,131.2,131.8,132.4,132.4,132.6,137.0,137.0,137.1$, 138.3, 165.4 .

Octamer 8. The compound (119 mg, $0.027 \mathrm{mmol}, 80 \%)$ was prepared from 14 (106 mg, $0.034 \mathrm{mmol})$ and $(P)-\mathbf{1}$ (54 mg, $0.069 \mathrm{mmol}$ ). Mp $248-254{ }^{\circ} \mathrm{C}$ (toluene-methanol). $[\alpha]_{\mathrm{D}}{ }^{25}+4640$ (c 0.10 , trifluoromethylbenzene, observed within 30 min after dissolution). MALDI TOF-MS m/z Calcd for ${ }^{12} \mathrm{C}_{314}{ }^{13} \mathrm{C}_{3} \mathrm{H}_{298} \mathrm{O}_{14} \mathrm{Si}_{2}$ : 4387.2. Found: 4386.6. UV-vis (trifluoromethylbenzene, $1 \mu \mathrm{M}$, observed within $10 \mathrm{~min}$ after dissolution) $\lambda_{\max }(\varepsilon) 332 \mathrm{~nm}\left(3.1 \times 10^{5}\right)$. CD $\left(\mathrm{CHCl}_{3}, 5 \mu \mathrm{M}\right.$, observed $5 \mathrm{~min}$ after dissolution) $\lambda(\Delta \varepsilon) 325 \mathrm{~nm}(-1550), 367 \mathrm{~nm}(2290), 385 \mathrm{~nm}(1620), 392 \mathrm{~nm}$ (1800). IR (KBr) 2207, 2146, $1722 \mathrm{~cm}^{-1}$. Anal. $\left(\mathrm{C}_{317} \mathrm{H}_{298} \mathrm{O}_{14} \mathrm{Si}_{2}\right)$ Calcd for: C, 86.77; H, 6.85\%. Found: C, 86.38; $\mathrm{H}$, 6.94\%. ${ }^{1} \mathrm{H}$ NMR (600 MHz, $\mathrm{CDCl}_{3}, 1 \mathrm{mM}$, observed at $60{ }^{\circ} \mathrm{C}$ after being heated at $60{ }^{\circ} \mathrm{C}$ for $\left.2 \mathrm{~h}\right) \delta 0.37(18 \mathrm{H}, \mathrm{s})$, $0.85(21 \mathrm{H}, \mathrm{t}, J=7.2 \mathrm{~Hz}), 1.22-1.45(84 \mathrm{H}, \mathrm{m}), 1.45-1.53(14 \mathrm{H}, \mathrm{m}), 1.80-1.88(14 \mathrm{H}, \mathrm{m}), 1.94(6 \mathrm{H}, \mathrm{s}), 1.95(6 \mathrm{H}, \mathrm{s})$, $1.98(36 \mathrm{H}, \mathrm{s}), 4.40-4.43(14 \mathrm{H}, \mathrm{m}), 7.43(2 \mathrm{H}, \mathrm{d}, J=7.6 \mathrm{~Hz}), 7.45(2 \mathrm{H}, \mathrm{d}, J=7.6 \mathrm{~Hz}), 7.48(12 \mathrm{H}, \mathrm{d}, J=6.9 \mathrm{~Hz})$, $7.64(2 \mathrm{H}, \mathrm{dd}, J=7.6,8.9 \mathrm{~Hz}), 7.68-7.72(14 \mathrm{H}, \mathrm{m}), 8.00(2 \mathrm{H}, \mathrm{s}), 8.07(2 \mathrm{H}, \mathrm{s}), 8.12(4 \mathrm{H}, \mathrm{s}), 8.13(8 \mathrm{H}, \mathrm{s}), 8.16(2 \mathrm{H}, \mathrm{t}$, $J=1.7 \mathrm{~Hz}), 8.18(5 \mathrm{H}, \mathrm{s}), 8.32-8.33(2 \mathrm{H}, \mathrm{m}), 8.34-8.35(12 \mathrm{H}, \mathrm{m}), 8.42(2 \mathrm{H}, \mathrm{d}, J=8.9 \mathrm{~Hz}), 8.51-8.55(14 \mathrm{H}, \mathrm{m})$. ${ }^{13} \mathrm{C}$ NMR $\left(150 \mathrm{MHz}, \mathrm{CDCl}_{3}, 1 \mathrm{mM}\right.$, observed at $60{ }^{\circ} \mathrm{C}$ after being heated at $60{ }^{\circ} \mathrm{C}$ for $\left.2 \mathrm{~h}\right) \delta 0.13,14.0,14.0,22.7$, 23.1, 23.1, 23.1, 26.1, 28.8, 28.9, 29.3, 29.3, 29.6, 31.9, 31.9, 65.8, 89.5, 93.1, 120.1, 120.6, 123.7, 123.7, 123.7, $123.8,124.5,127.0,127.0,127.1,129.1,129.2,129.3,129.9,130.0,131.2,131.2,131.8,132.4,132.4,137.1,137.1$, $138.3,165.5$.

Nonamer 9. The compound (27 mg, $5.5 \mu \mathrm{mol}, 67 \%)$ was prepared from $15(30 \mathrm{mg}, 8.2 \mu \mathrm{mol})$ and $(P)-\mathbf{1}(13 \mathrm{mg}$, $0.017 \mathrm{mmol}$ ) as was 4 except that 15 was dissolved in tetrahydrofuran. Mp $257-259{ }^{\circ} \mathrm{C}$ (toluene-methanol). $[\alpha]_{\mathrm{D}}{ }^{24}$ +4704 ( $c$ 0.10, trifluoromethylbenzene, observed within $30 \mathrm{~min}$ after dissolution). MALDI TOF-MS $\mathrm{m} / \mathrm{z}$ Calcd for ${ }^{12} \mathrm{C}_{355}{ }^{13} \mathrm{C}_{3} \mathrm{H}_{336} \mathrm{O}_{16} \mathrm{Si}_{2}$ : 4949.5. Found: 4949.3. UV-vis (trifluoromethylbenzene, $1 \mu \mathrm{M}$, observed within $10 \mathrm{~min}$ after dissolution) $\lambda_{\max }(\varepsilon) 332 \mathrm{~nm}\left(3.3 \times 10^{5}\right) . \mathrm{CD}\left(\mathrm{CHCl}_{3}, 5 \mu \mathrm{M}\right.$, observed 5 min after dissolution $) \lambda(\Delta \varepsilon) 328 \mathrm{~nm}$ (-1960), $368 \mathrm{~nm}$ (2920), $385 \mathrm{~nm}$ (2090), $392 \mathrm{~nm}$ (2330). IR (KBr) 2207, 2147, $1721 \mathrm{~cm}^{-1}$. Anal. $\left(\mathrm{C}_{358} \mathrm{H}_{336} \mathrm{O}_{16} \mathrm{Si}_{2}\right)$ Calcd for: C, 86.85; H, 6.84\%. Found: C, 86.55; H, 7.08\%. ${ }^{1} \mathrm{H}$ NMR $\left(600 \mathrm{MHz}, \mathrm{CDCl}_{3}, 0.20 \mathrm{mM}\right.$, observed at $60{ }^{\circ} \mathrm{C}$ after being heated at $60{ }^{\circ} \mathrm{C}$ for $25 \mathrm{~h}$ ) gave broad signals. ${ }^{13} \mathrm{C}$ NMR $\left(150 \mathrm{MHz}, \mathrm{CDCl}_{3}, 0.97 \mathrm{mM}\right.$, observed at $60{ }^{\circ} \mathrm{C}$ after being heated at $60{ }^{\circ} \mathrm{C}$ for $\left.36 \mathrm{~h}\right) \delta 0.12,14.0,14.1,22.6,22.8,23.1,23.1,26.1,28.8,29.3,29.3,29.5$, 29.6, 29.7, 29.7, 29.7, 31.9, 32.0, 65.8, 89.4, 93.1, 120.0, 123.7, 124.5, 127.1, 129.3, 129.9, 131.1, 132.4, 137.1, $138.3,165.4$. 
The rate constant for unfolding of 7 in phenylacetylene.

(a)

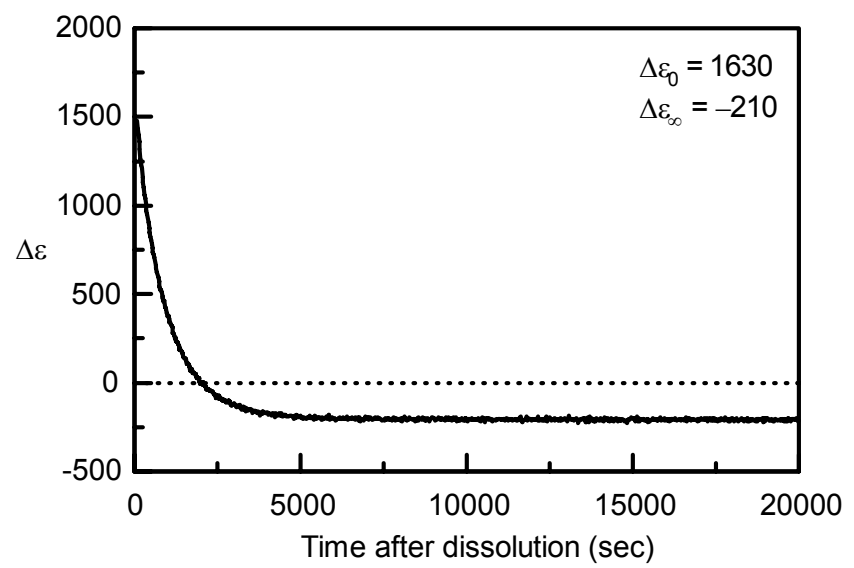

(b)

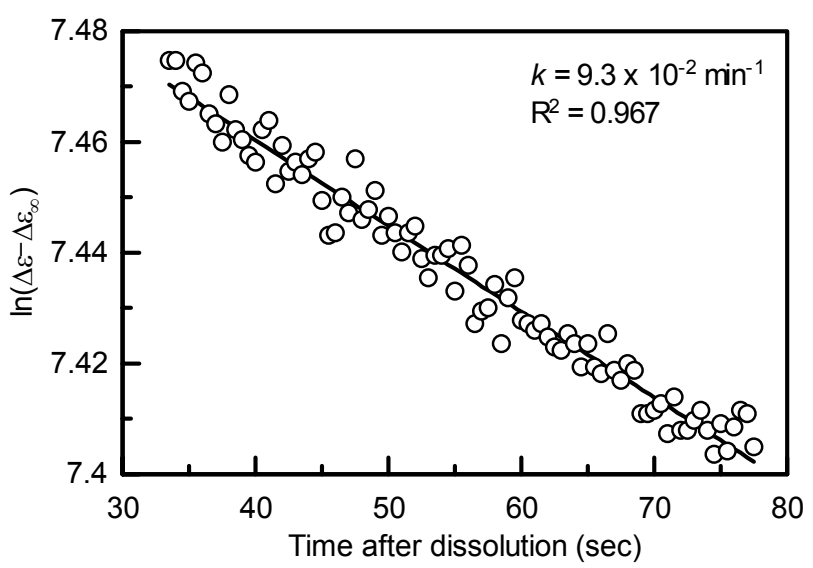

Figure S1. (a) Time dependence of $\Delta \varepsilon$ at $370 \mathrm{~nm}$ by $\mathrm{CD}\left(5 \mu \mathrm{M}, 25^{\circ} \mathrm{C}\right)$ in unfolding of 7 after dissolution in phenylacetylene. $\Delta \varepsilon_{0}=1630$ and $\Delta \varepsilon_{\infty}=-210$ were obtained. (b) Plots of $\ln \left(\Delta \varepsilon-\Delta \varepsilon_{\infty}\right)$ of $7(5 \mu \mathrm{M})$ at less than $10 \%$ conversion versus time after dissolution in phenylacetylene at $25{ }^{\circ} \mathrm{C}$, which gave $k=9.3 \times 10^{-2} \mathrm{~min}^{-1}$. Reproducibility was confirmed by conducting the same experiment, giving $k=9.5 \times 10^{-2} \mathrm{~min}^{-1}$.

The rate constant for unfolding of 7 in ethylbenzene.

(a)

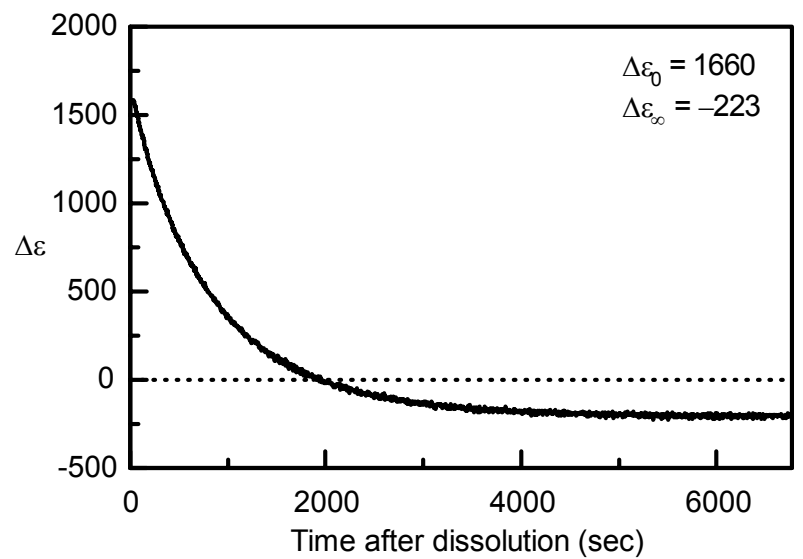

(b)

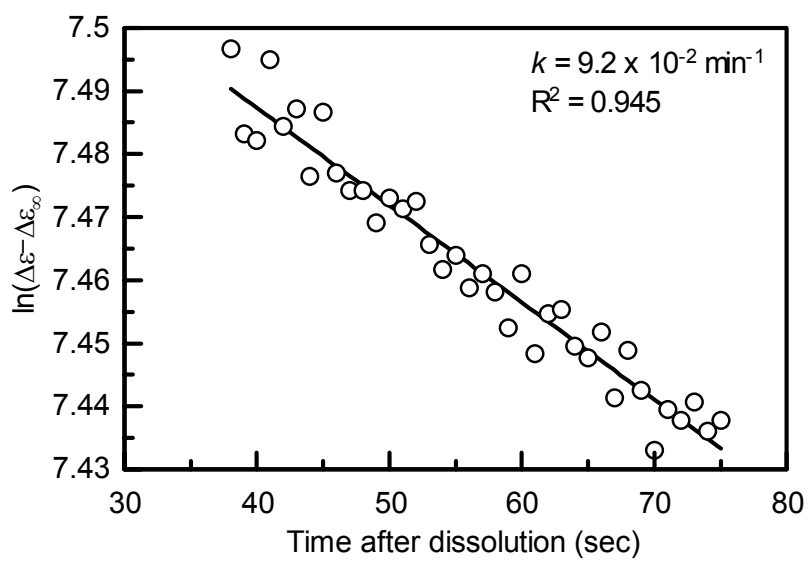

Figure S2. (a) Time dependence of $\Delta \varepsilon$ at $370 \mathrm{~nm}$ by $\mathrm{CD}\left(5 \mu \mathrm{M}, 25^{\circ} \mathrm{C}\right)$ in unfolding of 7 after dissolution in ethylbenzene. $\Delta \varepsilon_{0}=1660$ and $\Delta \varepsilon_{\infty}=-223$ were obtained. (b) Plots of $\ln \left(\Delta \varepsilon-\Delta \varepsilon_{\infty}\right)$ of 7 (5 $\left.\mu \mathrm{M}\right)$ at less than $10 \%$ conversion versus time after dissolution in ethylbenzene at $25{ }^{\circ} \mathrm{C}$, which gave $k=9.2 \times 10^{-2} \mathrm{~min}^{-1}$. Reproducibility was confirmed by conducting the same experiment, giving $k=9.0 \times 10^{-2} \mathrm{~min}^{-1}$. 
(a)

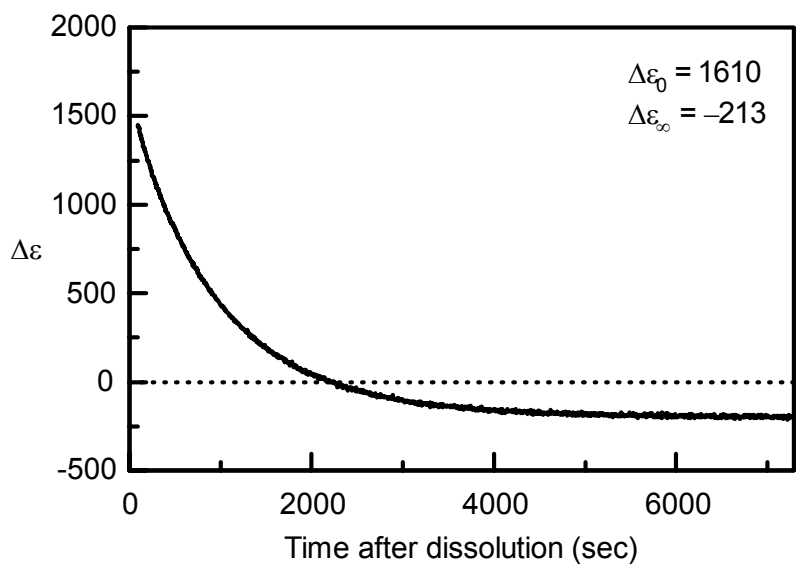

(b)

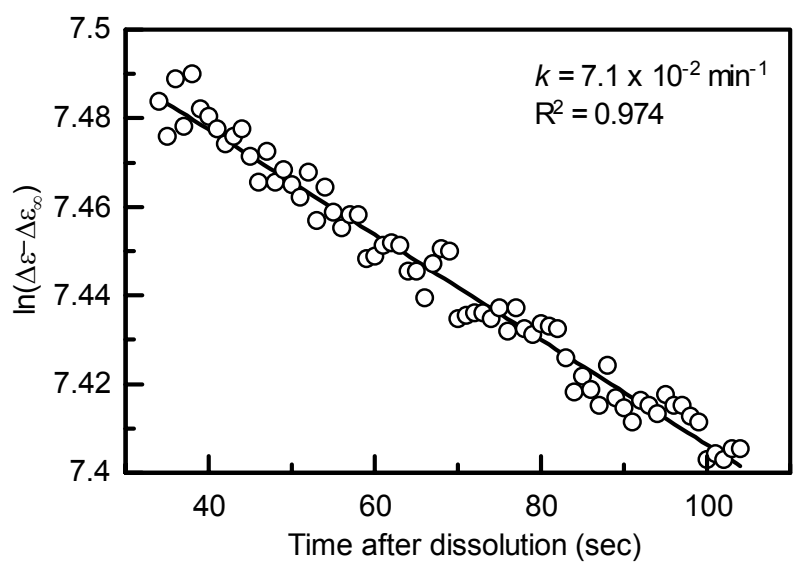

Figure S3. (a) Time dependence of $\Delta \varepsilon$ at $370 \mathrm{~nm}$ by $\mathrm{CD}\left(5 \mu \mathrm{M}, 25^{\circ} \mathrm{C}\right)$ in unfolding of 7 after dissolution in ethyl benzoate. $\Delta \varepsilon_{0}=1610$ and $\Delta \varepsilon_{\infty}=-213$ were obtained. (b) Plots of $\ln \left(\Delta \varepsilon-\Delta \varepsilon_{\infty}\right)$ of $7(5 \mu \mathrm{M})$ at less than $10 \%$ conversion versus time after dissolution in ethyl benzoate at $25{ }^{\circ} \mathrm{C}$, which gave $k=7.1 \times 10^{-2} \mathrm{~min}^{-1}$. Reproducibility was confirmed by conducting the same experiment, giving $k=7.6 \times 10^{-2} \mathrm{~min}^{-1}$. 
(a)

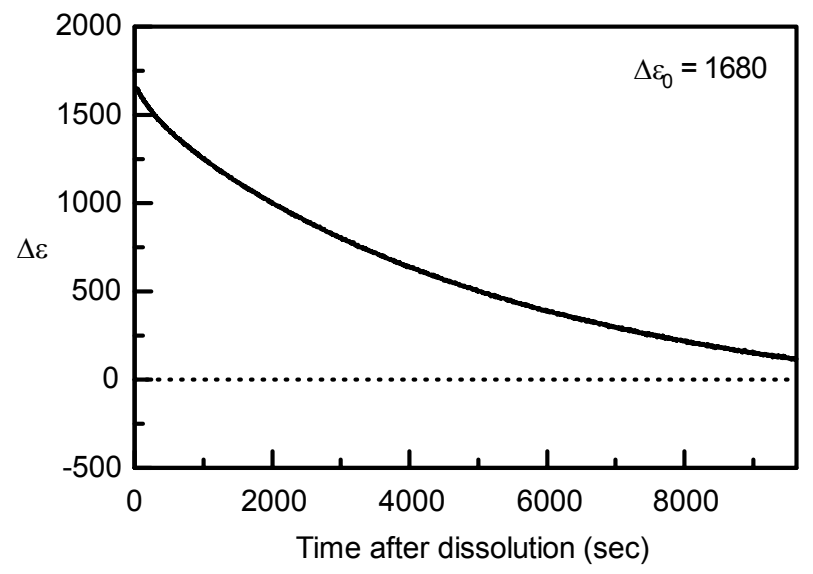

(c)

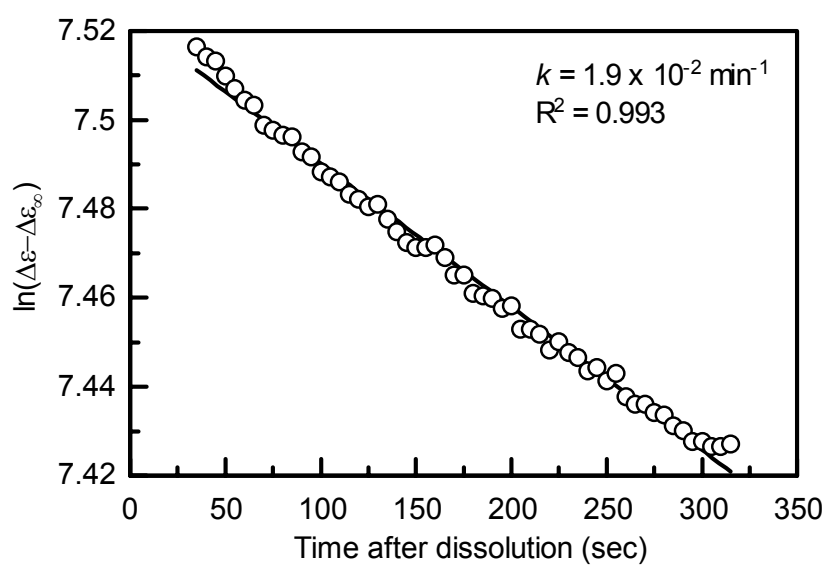

(b)

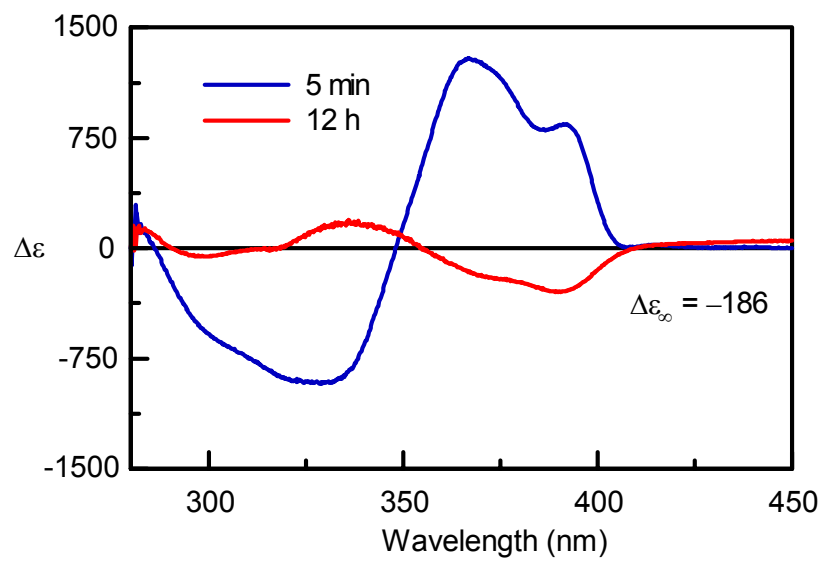

Figure S4. (a) Time dependence of $\Delta \varepsilon$ at $370 \mathrm{~nm}$ by $\mathrm{CD}\left(5 \mu \mathrm{M}, 25{ }^{\circ} \mathrm{C}\right)$ in unfolding of 7 after dissolution in toluene. $\Delta \varepsilon_{0}=1680$ was obtained. (b) $\mathrm{CD}$ spectra of $7\left(5 \mu \mathrm{M}, 25^{\circ} \mathrm{C}\right.$ ) at $5 \mathrm{~min}$ (blue line) and $12 \mathrm{~h}$ (red line) after dissolution in toluene, from which $\Delta \varepsilon_{\infty}=-186$ was obtained. (c) Plots of $\ln \left(\Delta \varepsilon-\Delta \varepsilon_{\infty}\right)$ of $7(5 \mu \mathrm{M})$ at less than $10 \%$ conversion versus time after dissolution in toluene at $25{ }^{\circ} \mathrm{C}$, which gave $k=1.9 \times 10^{-2} \mathrm{~min}^{-1}$. Reproducibility was confirmed by conducting the same experiment, giving $k=2.1 \times 10^{-2} \mathrm{~min}^{-1}$. 
The rate constant for unfolding of 7 in pyridine.

(a)

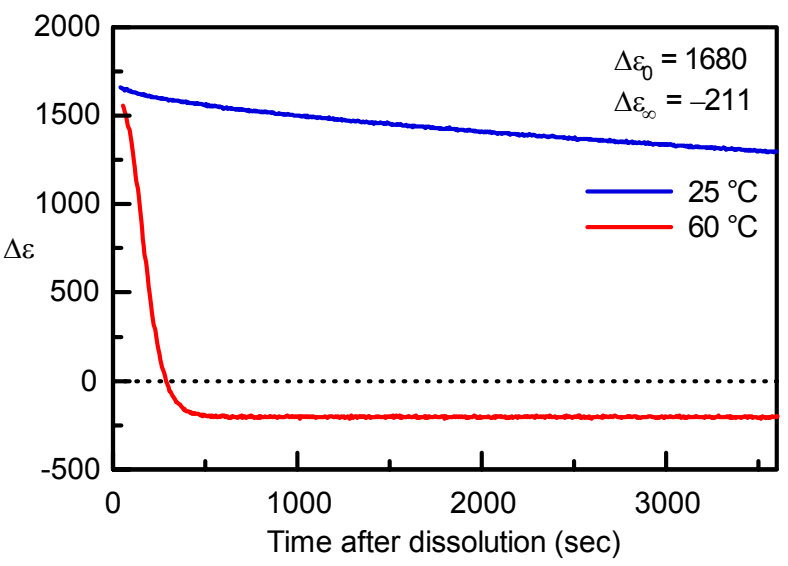

(b)

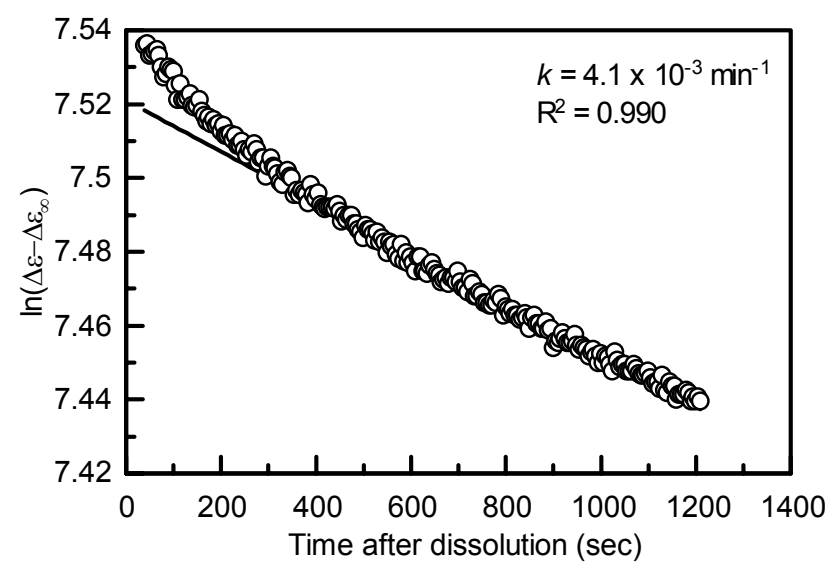

Figure S5. (a) Time dependences of $\Delta \varepsilon$ at $370 \mathrm{~nm}$ by $\mathrm{CD}(5 \mu \mathrm{M})$ in unfolding of 7 after dissolution in pyridine at $25^{\circ} \mathrm{C}$ (blue line) and $60{ }^{\circ} \mathrm{C}$ (red line). $\Delta \varepsilon_{0}=1680$ and $\Delta \varepsilon_{\infty}=-211$ were obtained. (b) Plots of $\ln \left(\Delta \varepsilon-\Delta \varepsilon_{\infty}\right)$ of 7 (5 $\mu \mathrm{M}$ ) at less than $10 \%$ conversion versus time after dissolution in pyridine at $25^{\circ} \mathrm{C}$, which gave $k=4.1 \times 10^{-3} \mathrm{~min}^{-1}$. Reproducibility was confirmed by conducting the same experiment, giving $k=4.3 \times 10^{-3} \mathrm{~min}^{-1}$. The deviation from straight at the initial stage may be due to the dissociation of intermolecular aggregation. 
(a)

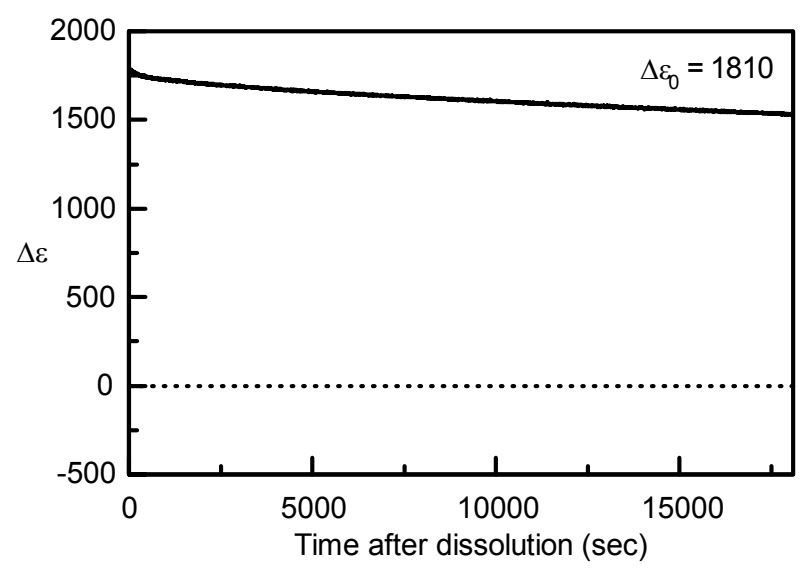

(c)

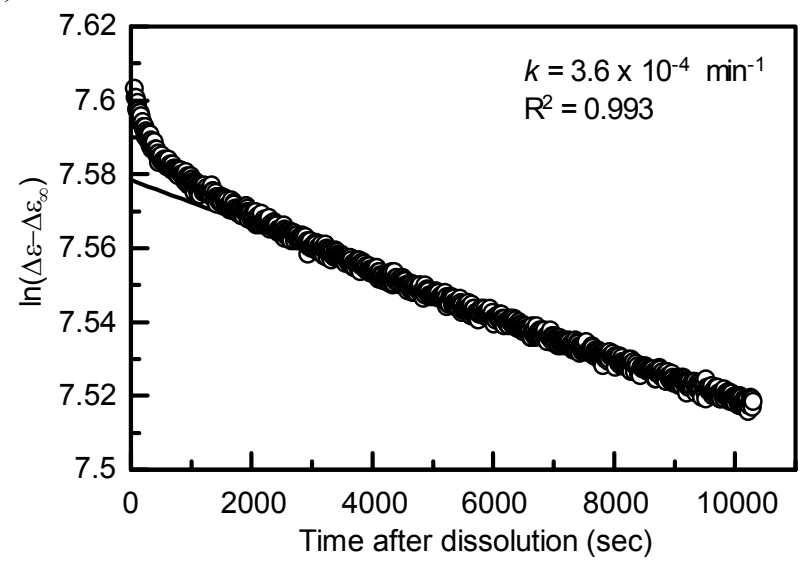

(b)

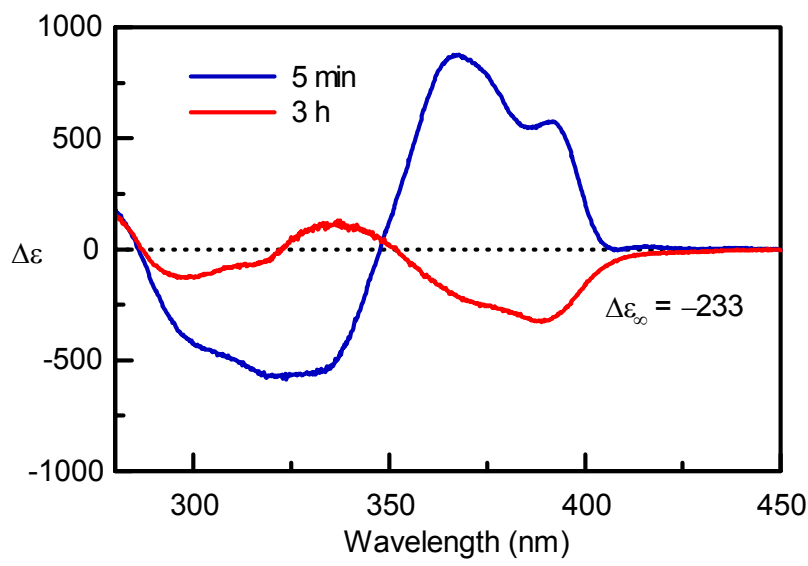

Figure S6. (a) Time dependence of $\Delta \varepsilon$ at $370 \mathrm{~nm}$ by $\mathrm{CD}\left(5 \mu \mathrm{M}, 25^{\circ} \mathrm{C}\right)$ in unfolding of 7 after dissolution in benzene. $\Delta \varepsilon_{0}=1810$ was obtained. (b) $\mathrm{CD}$ spectra of $7(5 \mu \mathrm{M})$ at $5 \mathrm{~min}$ (blue line) and $3 \mathrm{~h}$ (red line) after dissolution in benzene at $60{ }^{\circ} \mathrm{C}$, from which $\Delta \varepsilon_{\infty}=-233$ was obtained. (c) Plots of $\ln \left(\Delta \varepsilon-\Delta \varepsilon_{\infty}\right)$ of $7(5 \mu \mathrm{M})$ at less than $10 \%$ conversion versus time after dissolution in benzene at $25{ }^{\circ} \mathrm{C}$, which gave $k=3.6 \times 10^{-4} \mathrm{~min}^{-1}$. Reproducibility was confirmed by conducting the same experiment, giving $k=3.9 \times 10^{-4} \mathrm{~min}^{-1}$. The deviation from straight at the initial stage may be due to the dissociation of intermolecular aggregation. 
(a)

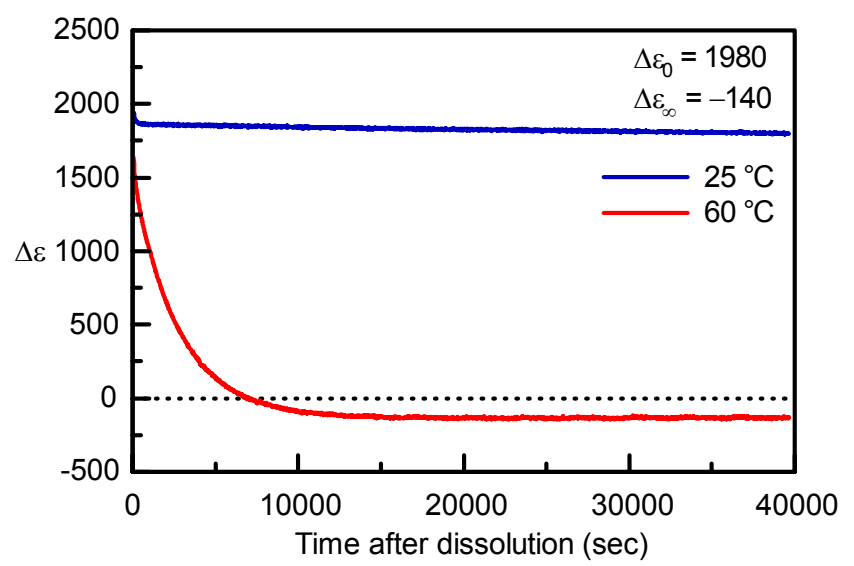

(b)

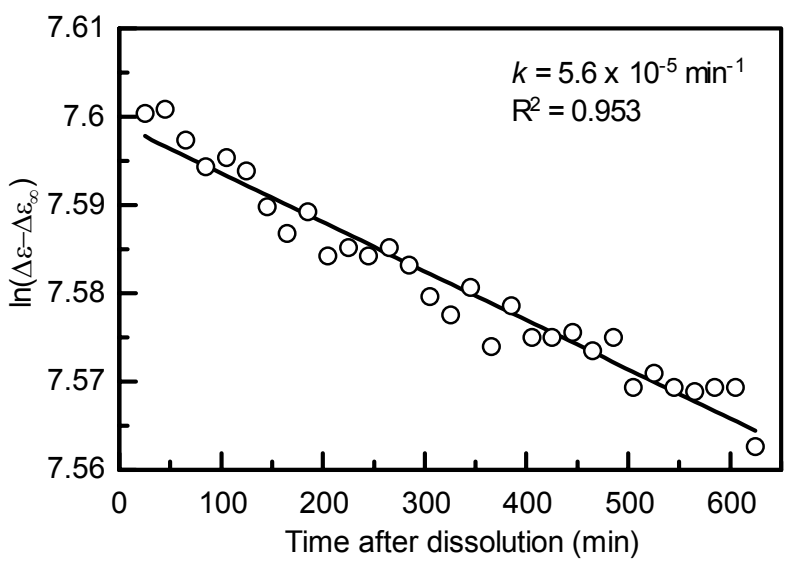

Figure S7. (a) Time dependences of $\Delta \varepsilon$ at $370 \mathrm{~nm}$ by $\mathrm{CD}(5 \mu \mathrm{M})$ in unfolding of 7 after dissolution in fluorobenzene at $25{ }^{\circ} \mathrm{C}$ (blue line) and $60{ }^{\circ} \mathrm{C}$ (red line). $\Delta \varepsilon_{0}=1980$ and $\Delta \varepsilon_{\infty}=-140$ were obtained. (b) Plots of $\ln \left(\Delta \varepsilon-\Delta \varepsilon_{\infty}\right)$ of $7(5 \mu \mathrm{M})$ at less than $10 \%$ conversion versus time after dissolution in fluorobenzene at $25{ }^{\circ} \mathrm{C}$, which gave $k=5.6 \times 10^{-5} \mathrm{~min}^{-1}$. Reproducibility was confirmed by conducting the same experiment, giving $k=5.7 \times 10^{-5}$ $\min ^{-1}$.

In styrene, thioanisole, bromobenzene, benzonitrile, anisole, and chlorobenzene, the unfolding was too fast at $25{ }^{\circ} \mathrm{C}$ to measure $\Delta \varepsilon$ at the conversion less than $10 \%$. The rate constants $k$ therefore were obtained at lower temperatures, and $k$ at $25{ }^{\circ} \mathrm{C}$ was extrapolated by the Arrenius plots.

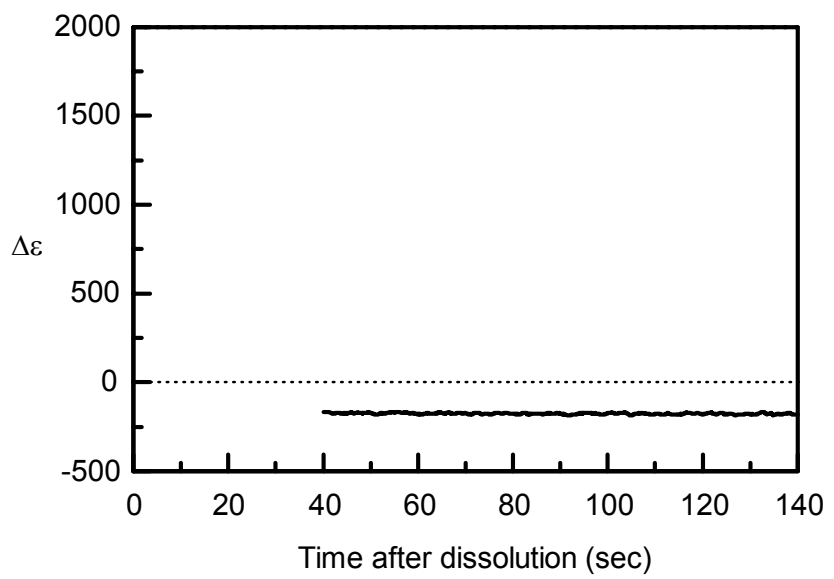

Figure S8. Time dependence of $\Delta \varepsilon$ at $370 \mathrm{~nm}\left(5 \mu \mathrm{M}, 25^{\circ} \mathrm{C}\right)$ in unfolding of 7 after dissolution in iodobenzene at $25^{\circ} \mathrm{C}$. The unfolding was complete within $40 \mathrm{sec}$. 
The rate constant for unfolding of 7 in styrene.

(a)

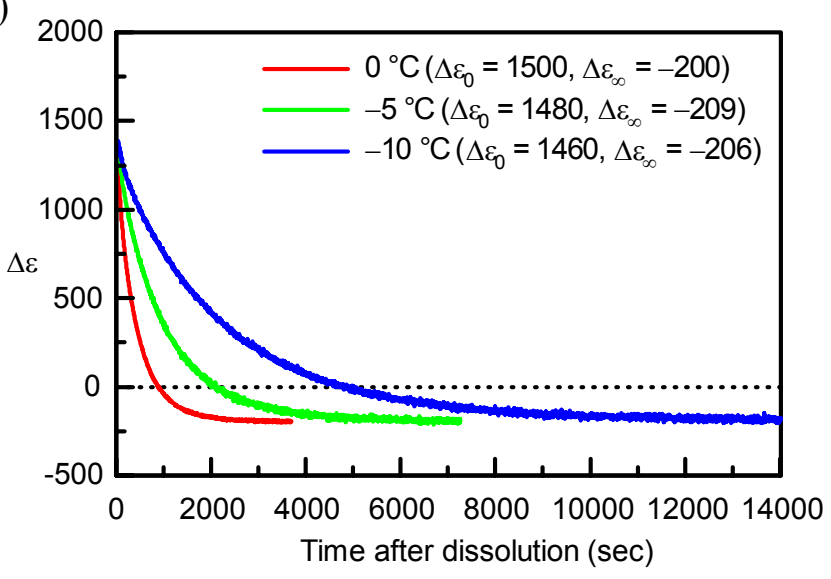

(c)

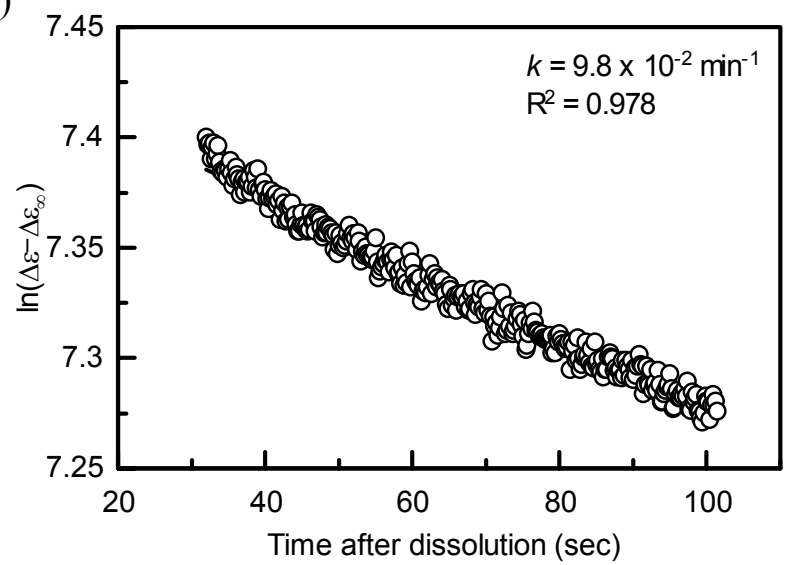

(b)

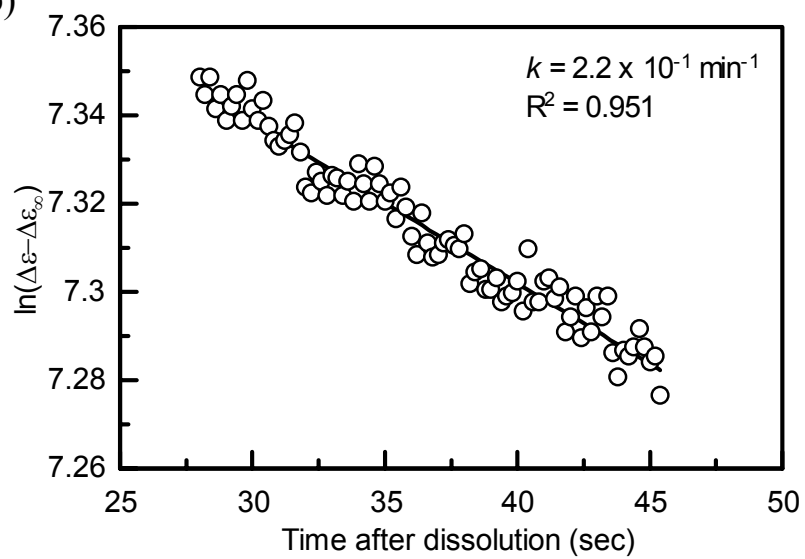

(d)

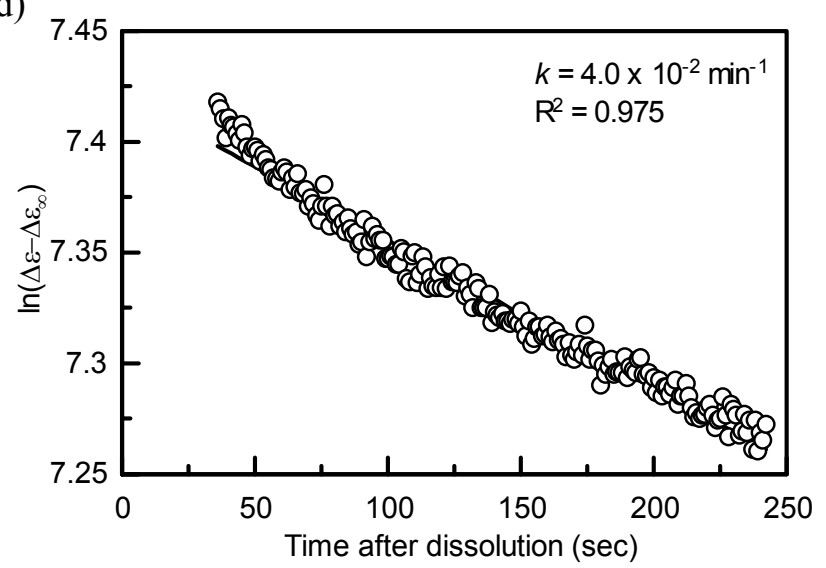

Figure S9. (a) Time dependences of $\Delta \varepsilon$ at $370 \mathrm{~nm}$ by $\mathrm{CD}(5 \mu \mathrm{M})$ in unfolding of 7 after dissolution in styrene at $0{ }^{\circ} \mathrm{C}$ (red line, $\left.\Delta \varepsilon_{0}=1500, \Delta \varepsilon_{\infty}=-200\right),-5{ }^{\circ} \mathrm{C}$ (green line, $\Delta \varepsilon_{0}=1480, \Delta \varepsilon_{\infty}=-209$ ), and $-10{ }^{\circ} \mathrm{C}$ (blue line, $\Delta \varepsilon_{0}=$ $\left.1460, \Delta \varepsilon_{\infty}=-206\right)$. Plots of $\ln \left(\Delta \varepsilon-\Delta \varepsilon_{\infty}\right)$ of $7(5 \mu \mathrm{M})$ at less than $15 \%$ conversion versus time after dissolution in styrene at $0{ }^{\circ} \mathrm{C}(\mathrm{b}),-5{ }^{\circ} \mathrm{C}(\mathrm{c})$, and $-10{ }^{\circ} \mathrm{C}(\mathrm{d})$, which gave $k=2.2 \times 10^{-1} \min ^{-1}\left(0{ }^{\circ} \mathrm{C}\right), 9.8 \times 10^{-2} \min ^{-1}\left(-5{ }^{\circ} \mathrm{C}\right)$, and $4.0 \times 10^{-2} \mathrm{~min}^{-1}\left(-10^{\circ} \mathrm{C}\right)$. Reproducibility was confirmed by conducting the same experiments, giving $k=2.3$ $\times 10^{-1} \min ^{-1}\left(0^{\circ} \mathrm{C}\right), 1.0 \times 10^{-1} \min ^{-1}\left(-5^{\circ} \mathrm{C}\right)$, and $4.2 \times 10^{-2} \min ^{-1}\left(-10^{\circ} \mathrm{C}\right)$.

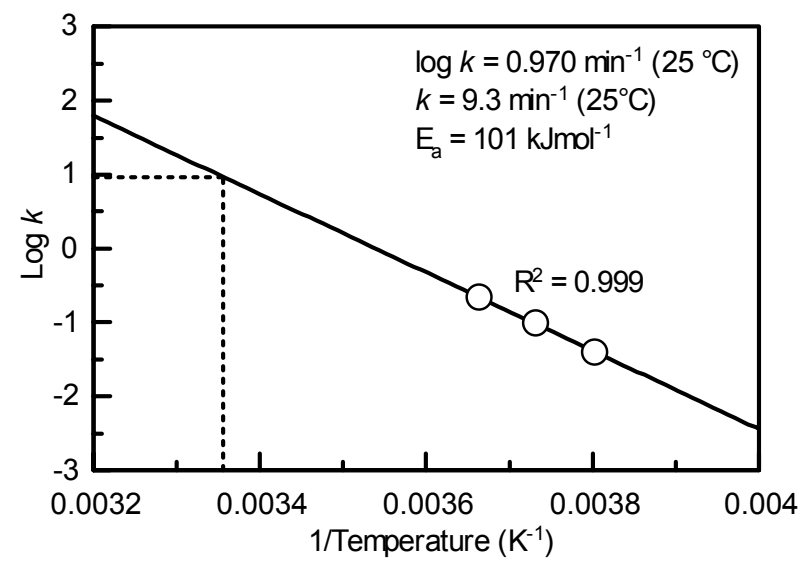

Figure S10. Estimation of $k=9.3 \mathrm{~min}^{-1}$ with activation energy $=101 \mathrm{kJmol}^{-1}$ in styrene at $25^{\circ} \mathrm{C}$ using Arrenius plots. Reproducibility was confirmed by conducting the same experiment, giving $k=9.8 \mathrm{~min}^{-1}$. 
The rate constant for unfolding of 7 in thioanisole.

(a)

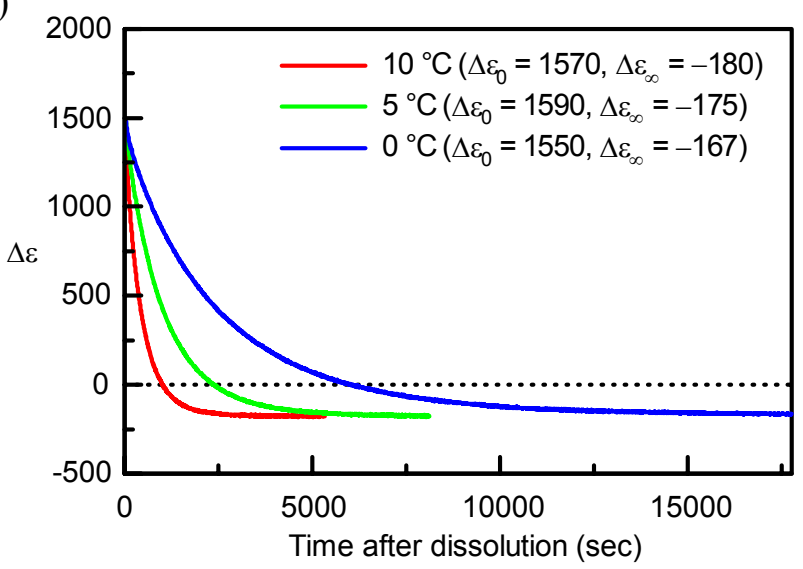

(c)

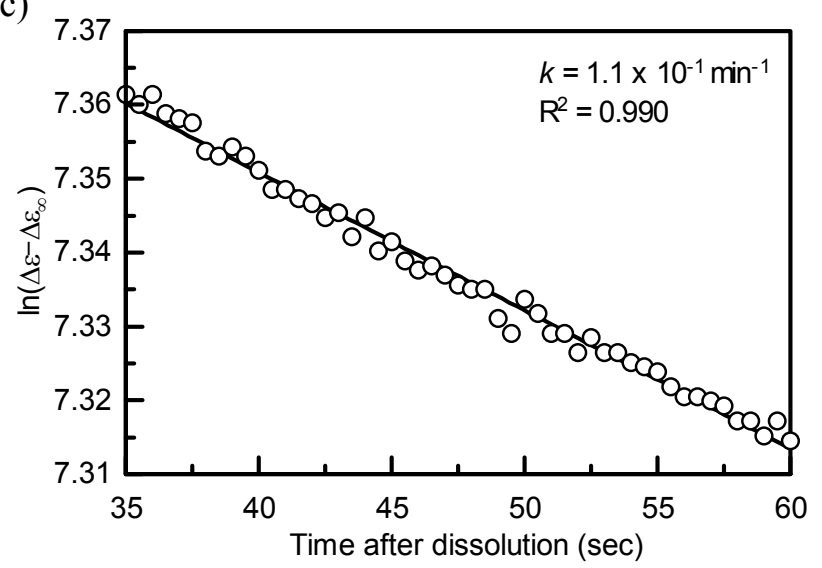

(b)

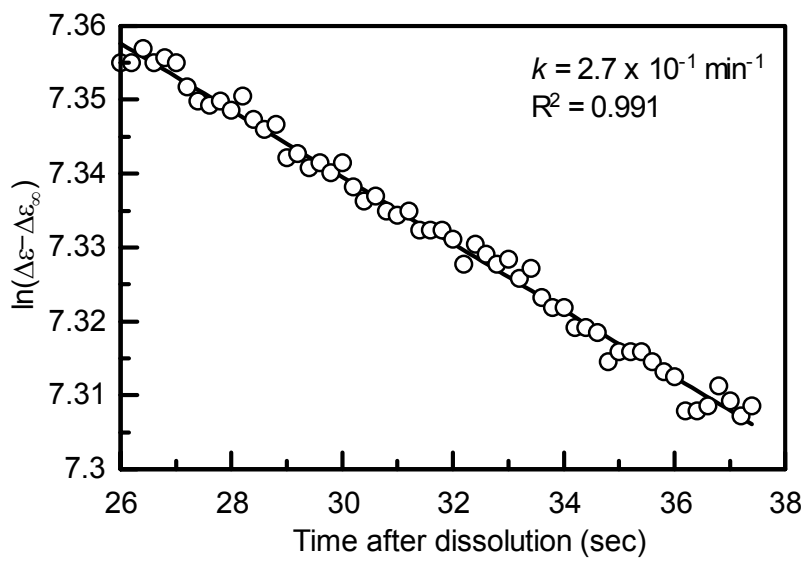

(d)

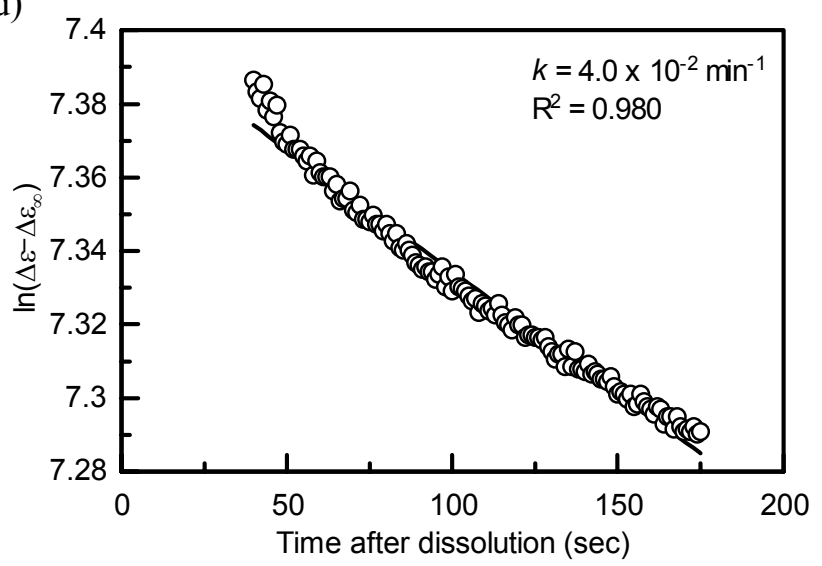

Figure S11. (a) Time dependences of $\Delta \varepsilon$ at $370 \mathrm{~nm}$ by $\mathrm{CD}(5 \mu \mathrm{M})$ in unfolding of 7 after dissolution in thioanisole at $10{ }^{\circ} \mathrm{C}$ (red line, $\Delta \varepsilon_{0}=1570, \Delta \varepsilon_{\infty}=-180$ ), $5{ }^{\circ} \mathrm{C}$ (green line, $\Delta \varepsilon_{0}=1590, \Delta \varepsilon_{\infty}=-175$ ), and $0{ }^{\circ} \mathrm{C}$ (blue line, $\Delta \varepsilon_{0}=$ $\left.1550, \Delta \varepsilon_{\infty}=-167\right)$. Plots of $\ln \left(\Delta \varepsilon-\Delta \varepsilon_{\infty}\right)$ of $7(5 \mu \mathrm{M})$ at less than $15 \%$ conversion versus time after dissolution in thioanisole at $10^{\circ} \mathrm{C}(\mathrm{b}), 5^{\circ} \mathrm{C}(\mathrm{c})$, and $0{ }^{\circ} \mathrm{C}(\mathrm{d})$, which gave $k=2.7 \times 10^{-1} \min ^{-1}\left(10^{\circ} \mathrm{C}\right), 1.1 \times 10^{-1} \min ^{-1}\left(5^{\circ} \mathrm{C}\right)$, and $4.0 \times 10^{-2} \mathrm{~min}^{-1}\left(0^{\circ} \mathrm{C}\right)$. Reproducibility was confirmed by conducting the same experiments, giving $k=2.7 \times 10^{-1}$ $\min ^{-1}\left(10^{\circ} \mathrm{C}\right), 1.1 \times 10^{-1} \min ^{-1}\left(5^{\circ} \mathrm{C}\right)$, and $3.3 \times 10^{-2} \min ^{-1}\left(0^{\circ} \mathrm{C}\right)$.

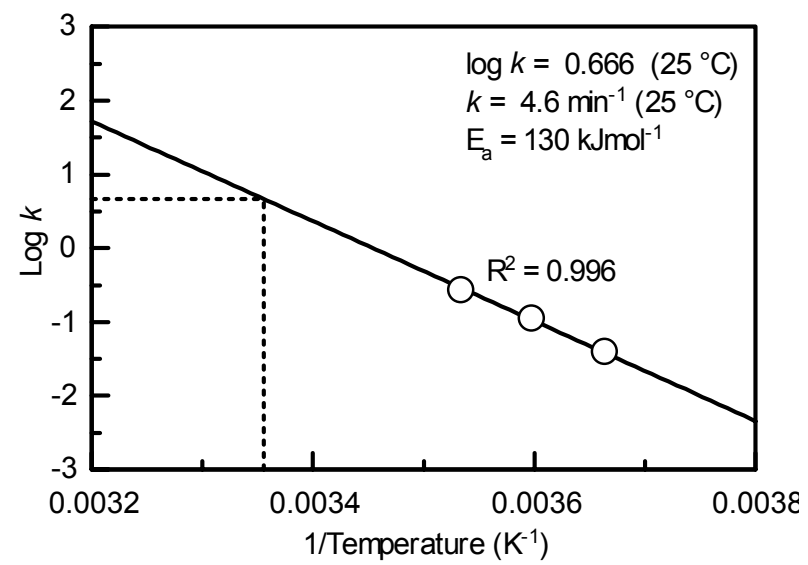

Figure S12. Estimation of $k=4.6 \mathrm{~min}^{-1}$ with activation energy $=130 \mathrm{kJmol}^{-1}$ in thioanisole at $25^{\circ} \mathrm{C}$ using Arrenius plots. Reproducibility was confirmed by conducting the same experiment, giving $k=5.1 \mathrm{~min}^{-1}$. 
The rate constant for unfolding of 7 in bromobenzene.

(a)

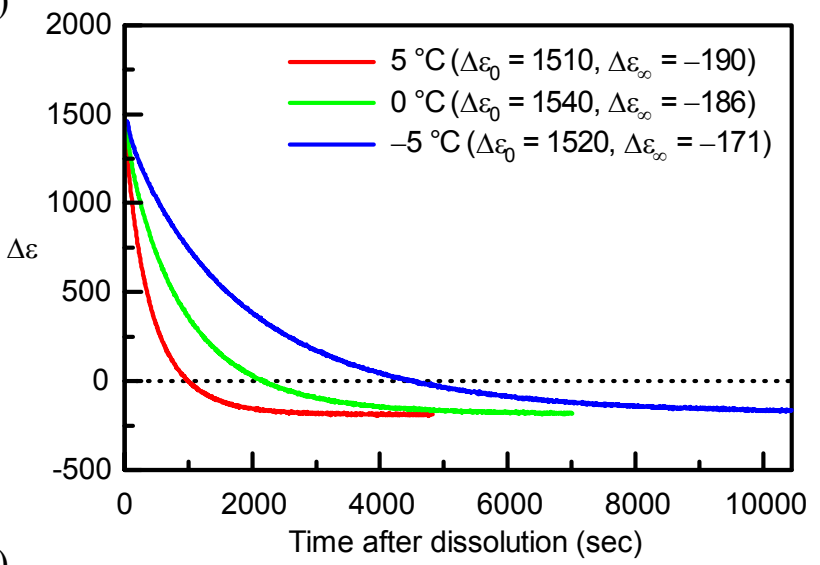

(c)

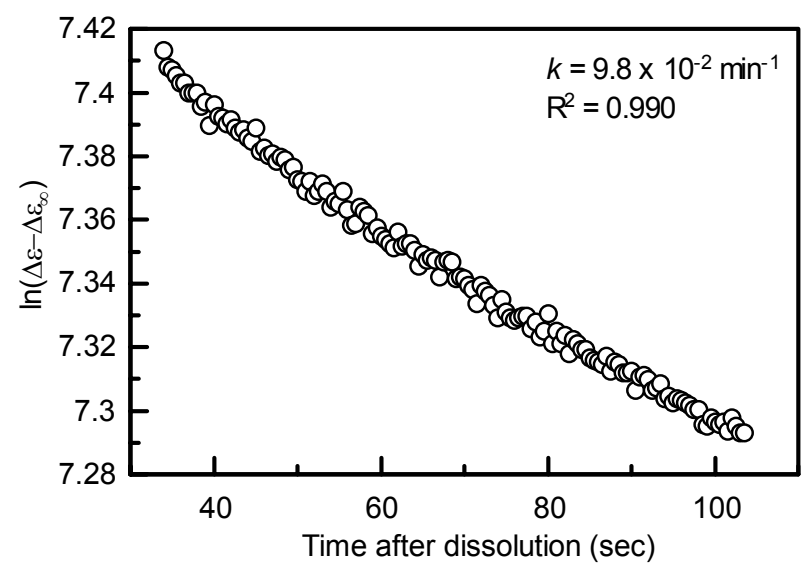

(b)

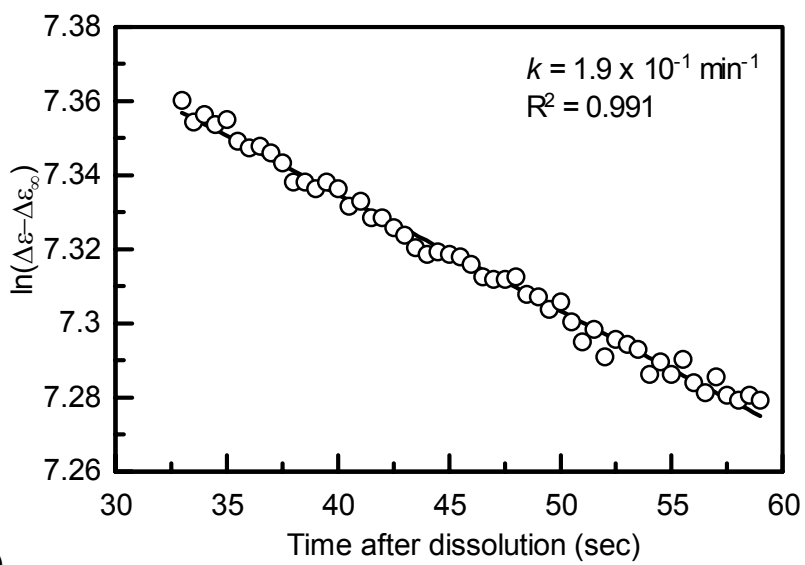

(d)

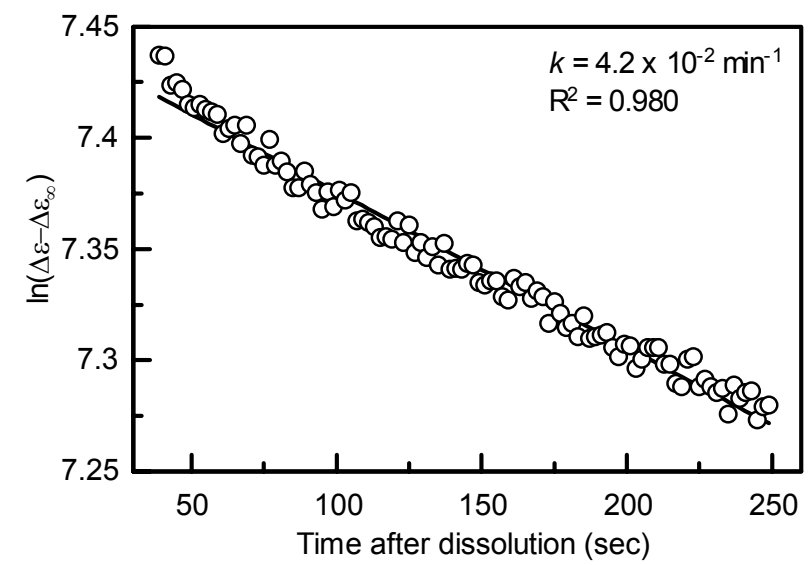

Figure S13. (a) Time dependences of $\Delta \varepsilon$ at $370 \mathrm{~nm}$ by $\mathrm{CD}(5 \mu \mathrm{M})$ in unfolding of 7 after dissolution in bromobenzene at $5{ }^{\circ} \mathrm{C}$ (red line, $\Delta \varepsilon_{0}=1510, \Delta \varepsilon_{\infty}=-190$ ), $0{ }^{\circ} \mathrm{C}$ (green, line, $\Delta \varepsilon_{0}=1540, \Delta \varepsilon_{\infty}=-186$ ), and $-5{ }^{\circ} \mathrm{C}$ (blue line, $\left.\Delta \varepsilon_{0}=1520, \Delta \varepsilon_{\infty}=-171\right)$. Plots of $\ln \left(\Delta \varepsilon-\Delta \varepsilon_{\infty}\right)$ of $7(5 \mu \mathrm{M})$ at conversion less than $15 \%$ versus time after dissolution in bromobenzene at less than $15 \%$ conversion at $5{ }^{\circ} \mathrm{C}(\mathrm{b}), 0{ }^{\circ} \mathrm{C}(\mathrm{c})$, and $-5{ }^{\circ} \mathrm{C}(\mathrm{d})$, which gave $k=1.9$ $\times 10^{-1} \min ^{-1}\left(5{ }^{\circ} \mathrm{C}\right), 9.8 \times 10^{-2} \min ^{-1}\left(0^{\circ} \mathrm{C}\right)$, and $4.2 \times 10^{-2} \min ^{-1}\left(-5^{\circ} \mathrm{C}\right)$. Reproducibility was confirmed by conducting the same experiments, giving $k=1.9 \times 10^{-1} \mathrm{~min}^{-1}\left(5^{\circ} \mathrm{C}\right), 1.1 \times 10^{-1} \min ^{-1}\left(0^{\circ} \mathrm{C}\right)$, and $4.6 \times 10^{-2} \min ^{-1}$ $\left(-5^{\circ} \mathrm{C}\right)$.

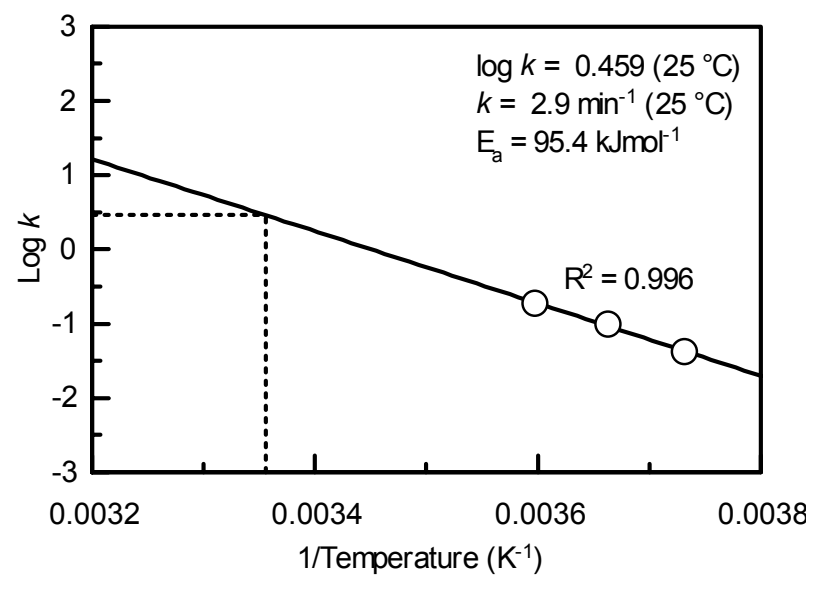

Figure S14. Estimation of $k=2.9 \mathrm{~min}^{-1}$ with activation energy $=95.4 \mathrm{kJmol}^{-1}$ in bromobenzene at $25{ }^{\circ} \mathrm{C}$ using Arrenius plots. Reproducibility was confirmed by conducting the same experiment, giving $k=2.5 \mathrm{~min}^{-1}$. 
The rate constant for unfolding of 7 in benzonitrile.

(a)

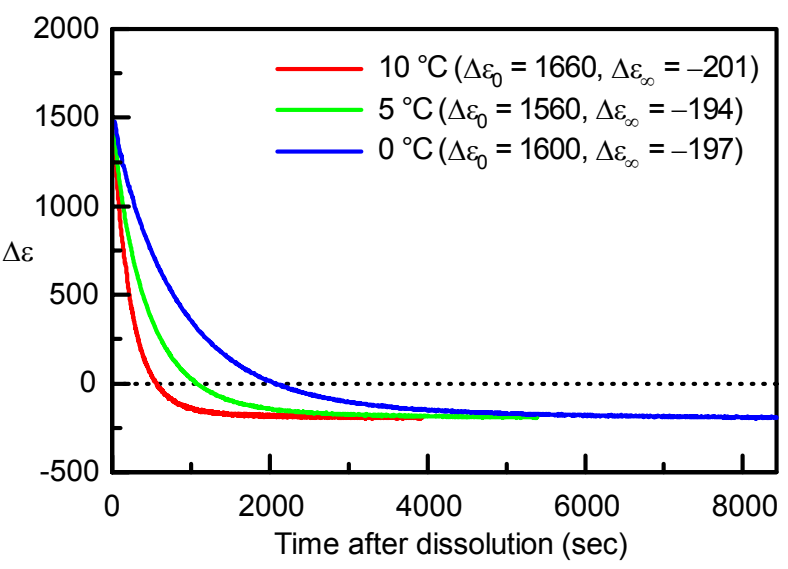

(c)

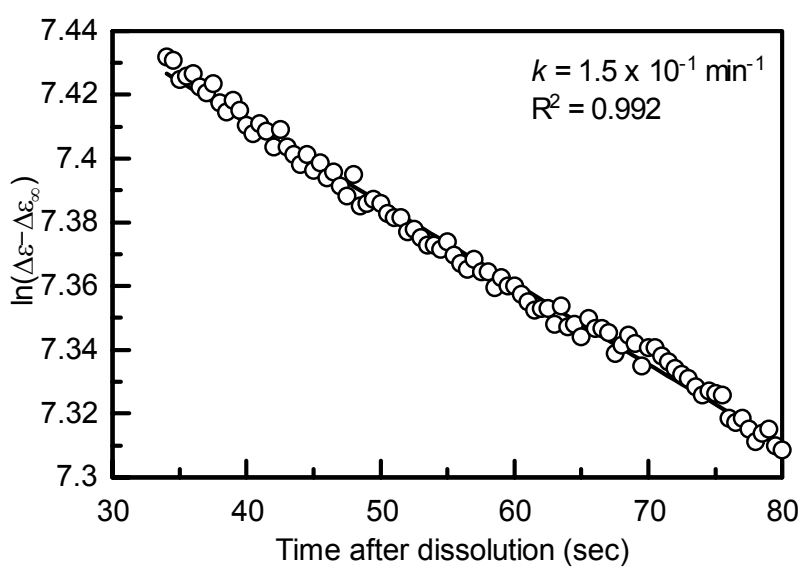

(b)

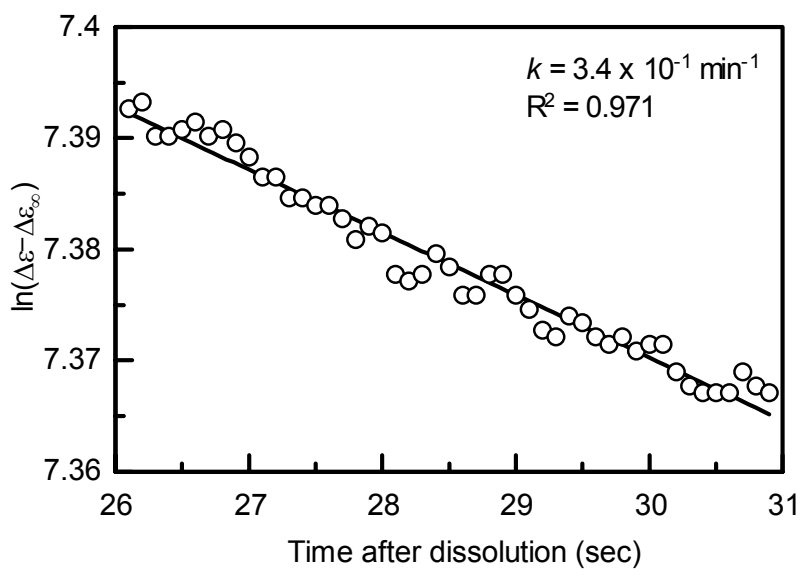

(d)

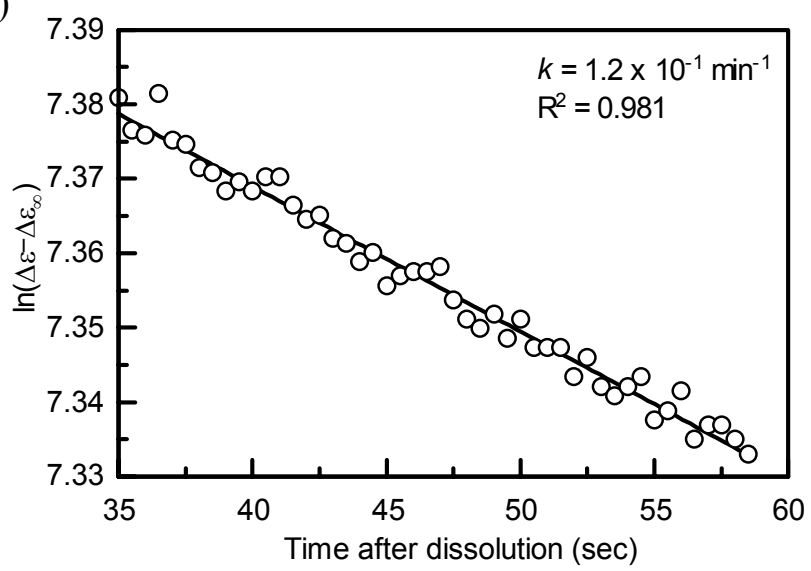

Figure S15. (a) Time dependences of $\Delta \varepsilon$ at $370 \mathrm{~nm}$ by $\mathrm{CD}(5 \mu \mathrm{M})$ in unfolding of 7 after dissolution in benzonitrile at $10{ }^{\circ} \mathrm{C}$ (red line, $\Delta \varepsilon_{0}=1660, \Delta \varepsilon_{\infty}=-201$ ), $5{ }^{\circ} \mathrm{C}$ (green line, $\Delta \varepsilon_{0}=1560, \Delta \varepsilon_{\infty}=-194$ ), and $0{ }^{\circ} \mathrm{C}$ (blue line, $\Delta \varepsilon_{0}=$ $\left.1600, \Delta \varepsilon_{\infty}=-197\right)$. Plots of $\ln \left(\Delta \varepsilon-\Delta \varepsilon_{\infty}\right)$ of $7(5 \mu \mathrm{M})$ at less than $15 \%$ conversion versus time after dissolution in benzonitrile at $10^{\circ} \mathrm{C}(\mathrm{b}), 5{ }^{\circ} \mathrm{C}(\mathrm{c})$, and $0^{\circ} \mathrm{C}(\mathrm{d})$, which gave $k=3.4 \times 10^{-1} \mathrm{~min}^{-1}\left(10^{\circ} \mathrm{C}\right), 1.5 \times 10^{-1} \min ^{-1}\left(5^{\circ} \mathrm{C}\right)$, and $1.2 \times 10^{-1} \mathrm{~min}^{-1}\left(0^{\circ} \mathrm{C}\right)$. Reproducibility was confirmed by conducting the same experiments, giving $k=3.0$ $\times 10^{-1} \min ^{-1}\left(10^{\circ} \mathrm{C}\right), 1.6 \times 10^{-1} \min ^{-1}\left(5^{\circ} \mathrm{C}\right)$, and $9.7 \times 10^{-2} \min ^{-1}\left(0^{\circ} \mathrm{C}\right)$.

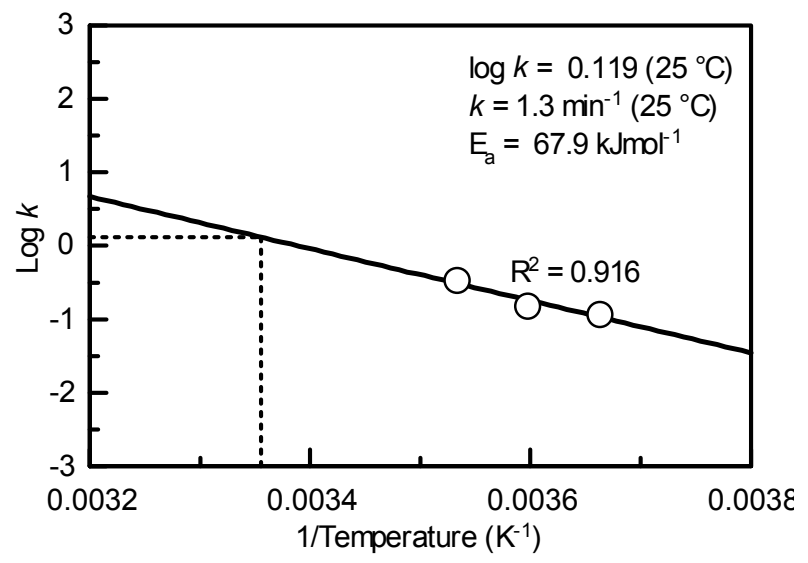

Figure S16. Estimation of $k=1.3 \mathrm{~min}^{-1}$ with activation energy $=67.9 \mathrm{kJmol}^{-1}$ in benzonitrile at $25{ }^{\circ} \mathrm{C}$ using Arrenius plots. Reproducibility was confirmed by conducting the same experiment, giving $k=1.3 \mathrm{~min}^{-1}$. 
(a)

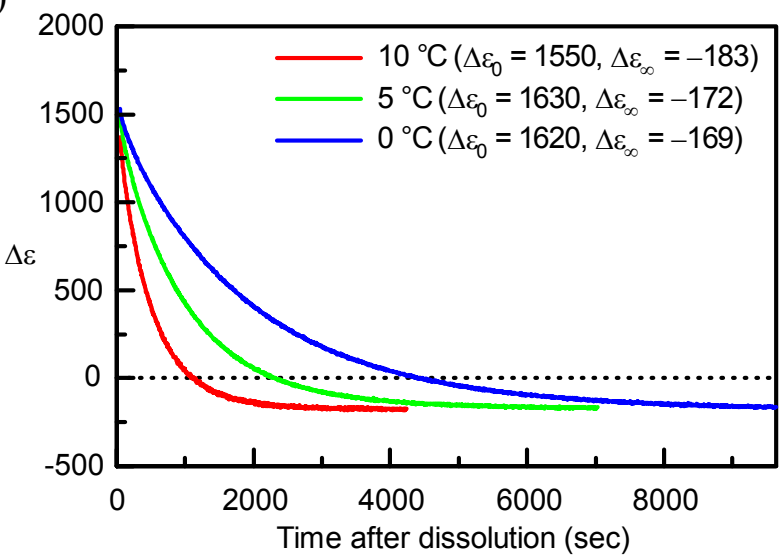

(c)

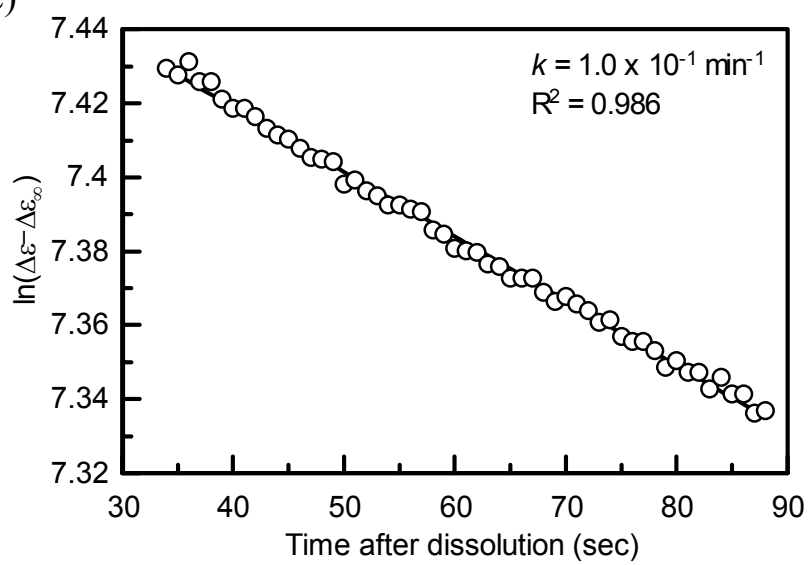

(b)

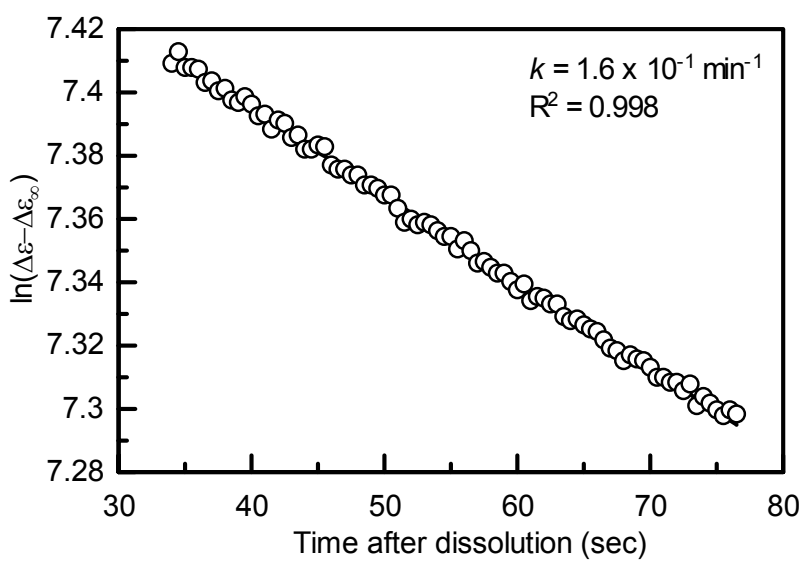

(d)

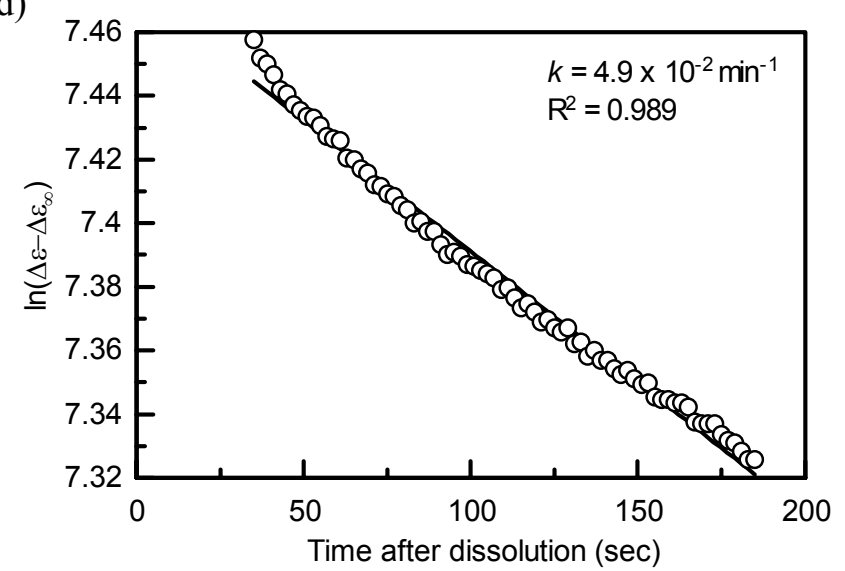

Figure S17. (a) Time dependences of $\Delta \varepsilon$ at $370 \mathrm{~nm}$ by $\mathrm{CD}(5 \mu \mathrm{M})$ in unfolding of 7 after dissolution in anisole at $10{ }^{\circ} \mathrm{C}$ (red line, $\Delta \varepsilon_{0}=1550, \Delta \varepsilon_{\infty}=-183$ ), $5{ }^{\circ} \mathrm{C}$ (green line, $\Delta \varepsilon_{0}=1630, \Delta \varepsilon_{\infty}=-172$ ), and $0{ }^{\circ} \mathrm{C}$ (blue line, $\Delta \varepsilon_{0}=$ $\left.1620, \Delta \varepsilon_{\infty}=-169\right)$. Plots of $\ln \left(\Delta \varepsilon-\Delta \varepsilon_{\infty}\right)$ of $7(5 \mu \mathrm{M})$ at less than $15 \%$ conversion versus time after dissolution in anisole at $10^{\circ} \mathrm{C}(\mathrm{b}), 5{ }^{\circ} \mathrm{C}(\mathrm{c})$, and $0{ }^{\circ} \mathrm{C}(\mathrm{d})$, which gave $k=1.6 \times 10^{-1} \mathrm{~min}^{-1}\left(10^{\circ} \mathrm{C}\right), 1.0 \times 10^{-1} \mathrm{~min}^{-1}\left(5^{\circ} \mathrm{C}\right)$, and 4.9 $\times 10^{-2} \mathrm{~min}^{-1}\left(0^{\circ} \mathrm{C}\right)$. Reproducibility was confirmed by conducting the same experiments, giving $k=1.7 \times 10^{-1} \mathrm{~min}^{-1}$ $\left(10^{\circ} \mathrm{C}\right), 1.2 \times 10^{-1} \min ^{-1}\left(5^{\circ} \mathrm{C}\right)$, and $5.4 \times 10^{-2} \min ^{-1}\left(0^{\circ} \mathrm{C}\right)$.

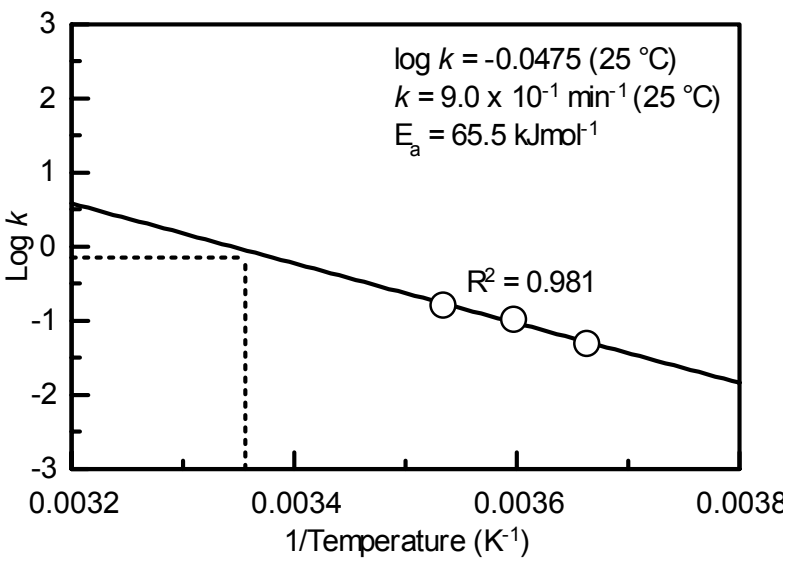

Figure S18. Estimation of $k=9.0 \times 10^{-1} \mathrm{~min}^{-1}$ with activation energy $=65.5 \mathrm{kJmol}^{-1}$ in anisole at $25{ }^{\circ} \mathrm{C}$ using Arrenius plots. Reproducibility was confirmed by conducting the same experiment, giving $k=8.2 \times 10^{-1} \mathrm{~min}^{-1}$. 
(a)

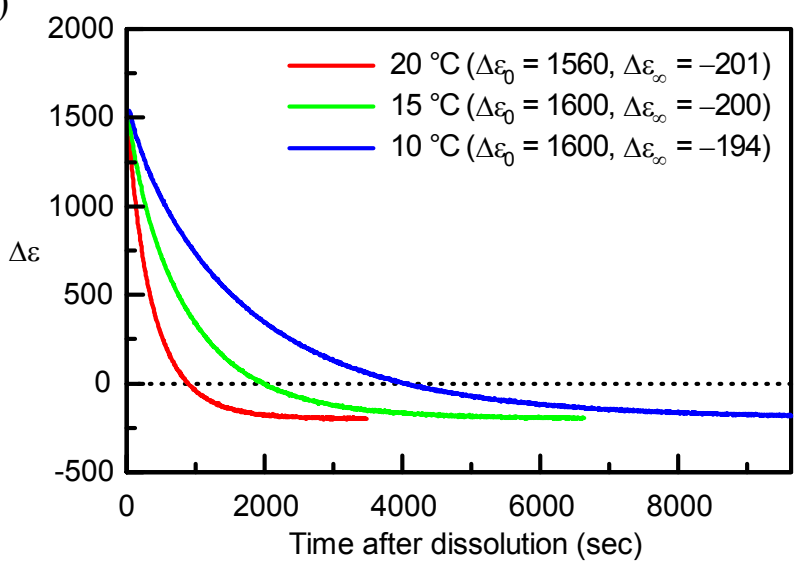

(c)

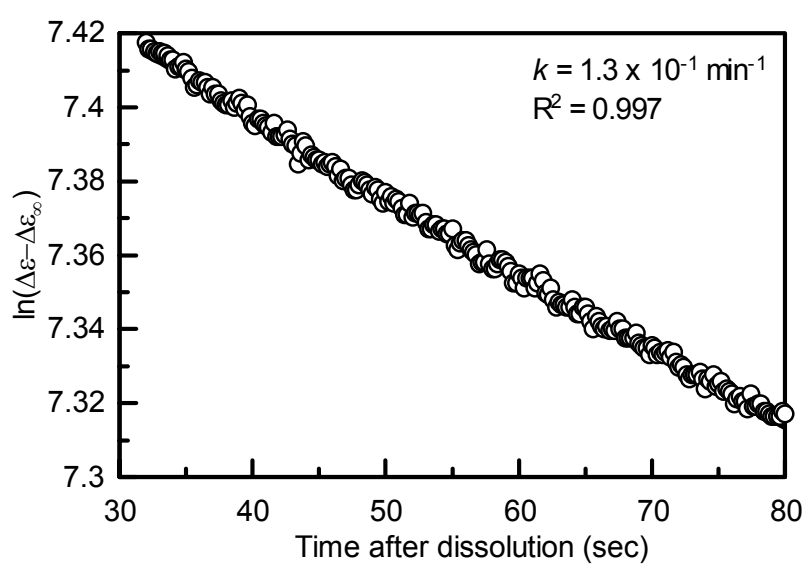

(b)

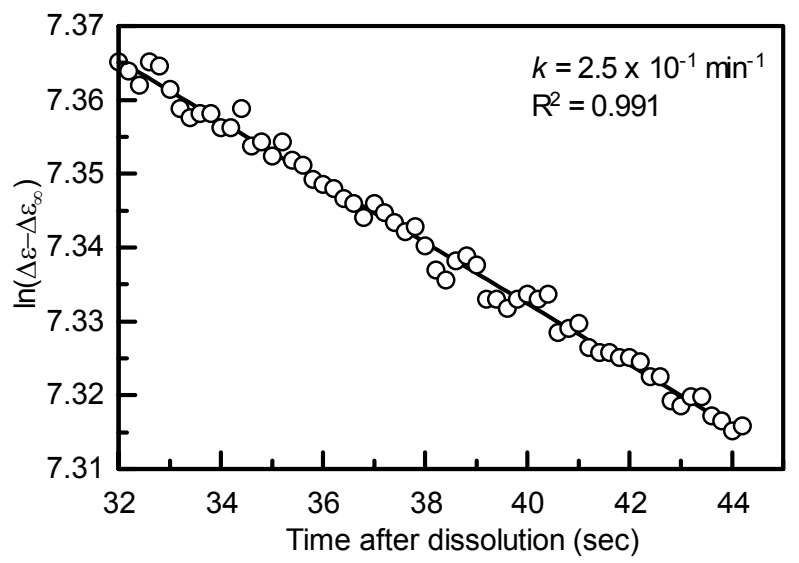

(d)

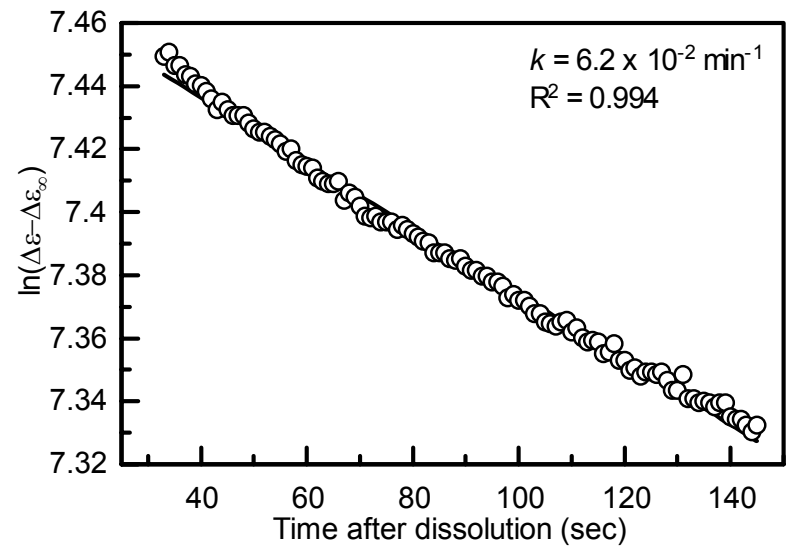

Figure S19. (a) Time dependences of $\Delta \varepsilon$ at $370 \mathrm{~nm}$ by $\mathrm{CD}(5 \mu \mathrm{M})$ in unfolding of 7 after dissolution in chlorobenzene at $20^{\circ} \mathrm{C}$ (red line, $\Delta \varepsilon_{0}=1560, \Delta \varepsilon_{\infty}=-201$ ), $15{ }^{\circ} \mathrm{C}$ (green line, $\Delta \varepsilon_{0}=1600, \Delta \varepsilon_{\infty}=-200$ ), and $10{ }^{\circ} \mathrm{C}$ (blue line, $\left.\Delta \varepsilon_{0}=1600, \Delta \varepsilon_{\infty}=-194\right)$. Plots of $\ln \left(\Delta \varepsilon-\Delta \varepsilon_{\infty}\right)$ of $7(5 \mu \mathrm{M})$ at less than $15 \%$ conversion versus time after dissolution in chlorobenzene at $20^{\circ} \mathrm{C}(\mathrm{b}), 15{ }^{\circ} \mathrm{C}(\mathrm{c})$, and $10{ }^{\circ} \mathrm{C}(\mathrm{d})$, which gave $k=2.5 \times 10^{-1} \mathrm{~min}^{-1}\left(20{ }^{\circ} \mathrm{C}\right), 1.3$ $\times 10^{-1} \min ^{-1}\left(15^{\circ} \mathrm{C}\right)$, and $6.2 \times 10^{-2} \min ^{-1}\left(10^{\circ} \mathrm{C}\right)$. Reproducibility was confirmed by conducting the same experiments, giving $k=2.6 \times 10^{-1} \min ^{-1}\left(20^{\circ} \mathrm{C}\right), 1.2 \times 10^{-1} \min ^{-1}\left(15^{\circ} \mathrm{C}\right)$, and $5.8 \times 10^{-2} \min ^{-1}\left(10^{\circ} \mathrm{C}\right)$.

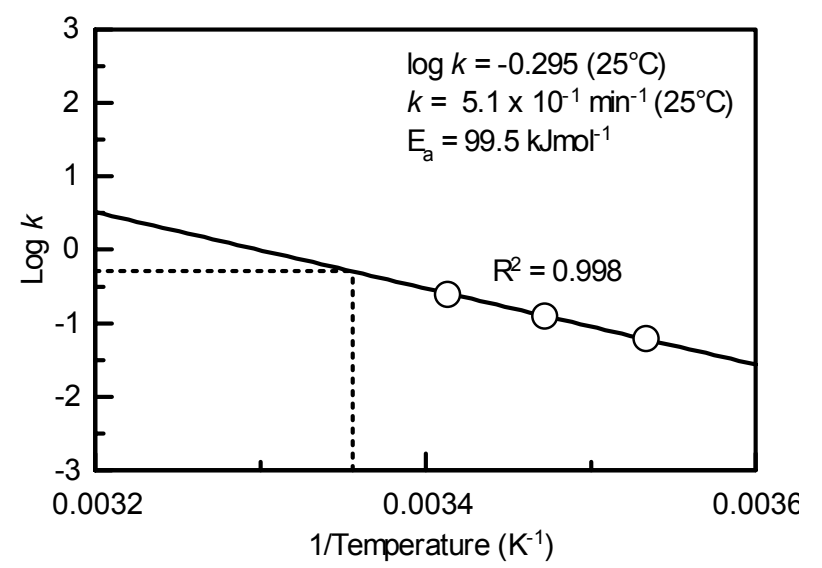

Figure S20. Estimation of $k=5.1 \times 10^{-1} \mathrm{~min}^{-1}$ with activation energy $=99.5 \mathrm{kJmol}^{-1}$ in chlorobenzene at $25{ }^{\circ} \mathrm{C}$ using Arrenius plots. Reproducibility was confirmed by conducting the same experiment, giving $k=5.1 \times 10^{-1}$ $\min ^{-1}$. 


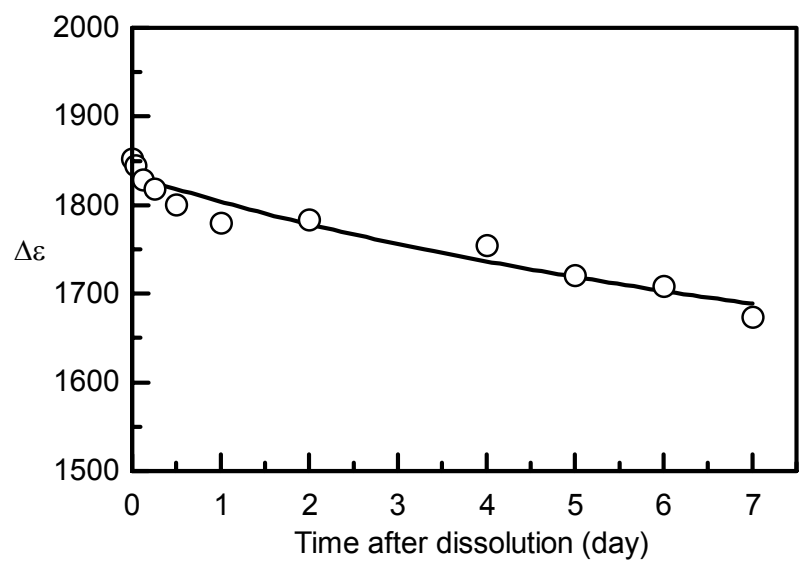

Figure S21. Time dependence of $\Delta \varepsilon$ at $370 \mathrm{~nm}\left(5 \mu \mathrm{M}, 25{ }^{\circ} \mathrm{C}\right)$ in unfolding of 7 after dissolution in $m$-difluorobenzene at $25^{\circ} \mathrm{C}$. The line was drawn to guide the eye. 
VPO Studies. The VPO analyses of helical 7 in benzene (Figure S22) and fluorobenzene (Figure S23) were conducted at $5 \mathrm{mM}$. The $\mathrm{CD}$ analyses revealed that the helical structures were maintained for several hours in both solvents at $1 \mathrm{mM}$. The VPO analyses of unfolded 7 were conducted in toluene at several concentrations (Figure S24). The CD spectrum of unfolded 7 in toluene $(1 \mathrm{mM})$ after being heated at $40{ }^{\circ} \mathrm{C}$ for $3 \mathrm{~h}$ was obtained to confirm the random coil formation.

(a)

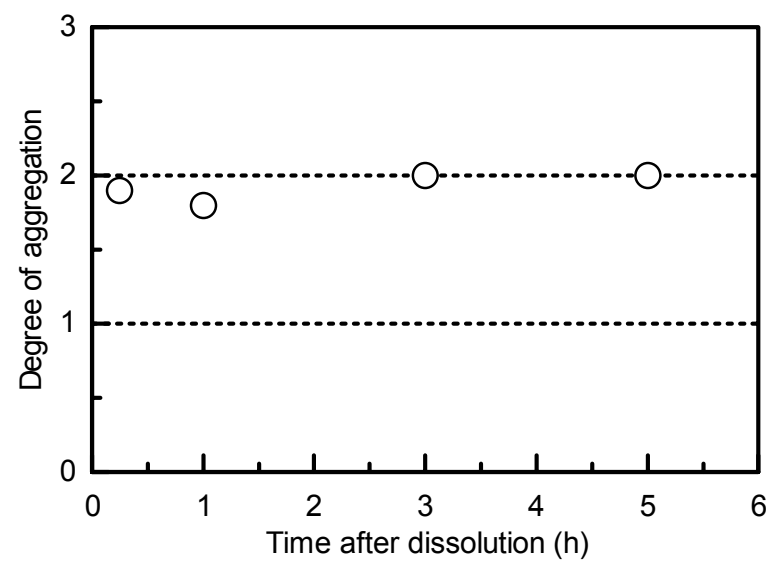

(b)

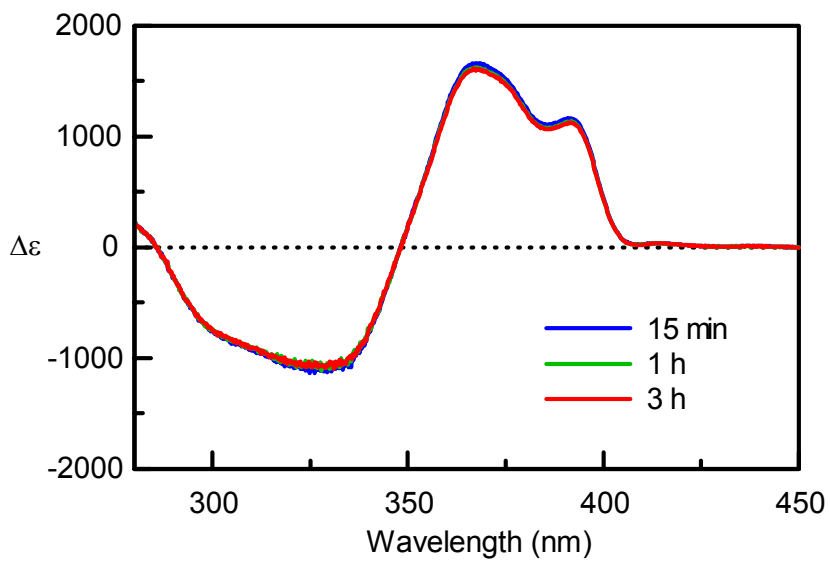

Figure S22. (a) Degree of aggregation of 7 by VPO $\left(5 \mathrm{mM}, 30^{\circ} \mathrm{C}\right)$ at various times after dissolution in benzene. (b) CD spectra of $7\left(1 \mathrm{mM}, 30^{\circ} \mathrm{C}\right)$ at various times after dissolution in benzene.

(a)

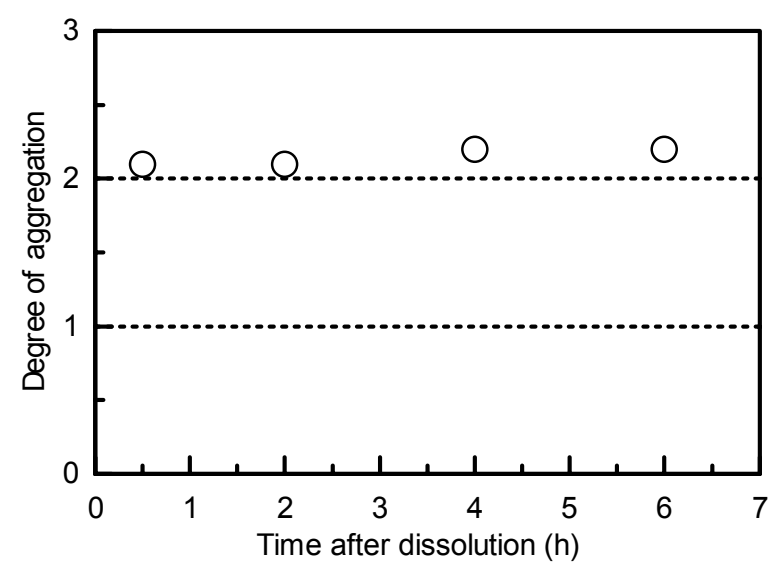

(b)

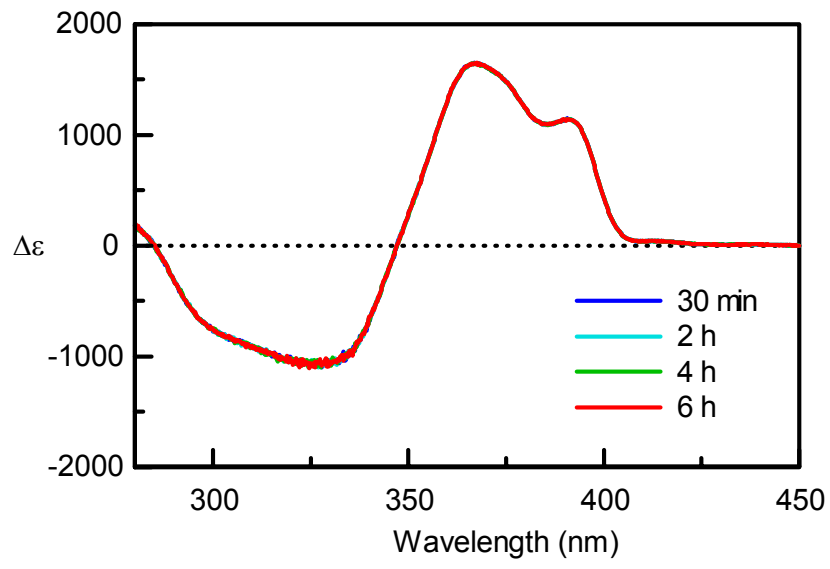

Figure S23. (a) Degree of aggregation of 7 by VPO $\left(5 \mathrm{mM}, 40{ }^{\circ} \mathrm{C}\right)$ at various times after dissolution in fluorobenzene. (b) CD spectra of $7\left(1 \mathrm{mM}, 40^{\circ} \mathrm{C}\right)$ at various times after dissolution in fluorobenzene. 
(a)

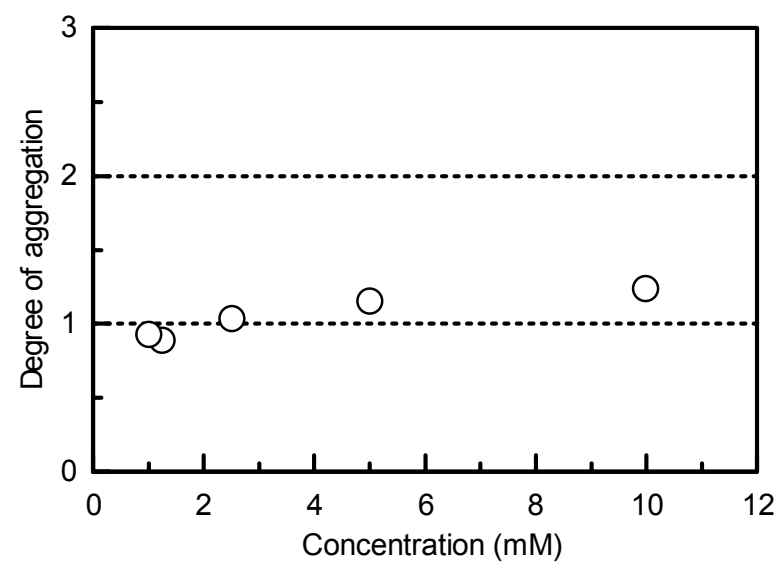

(b)

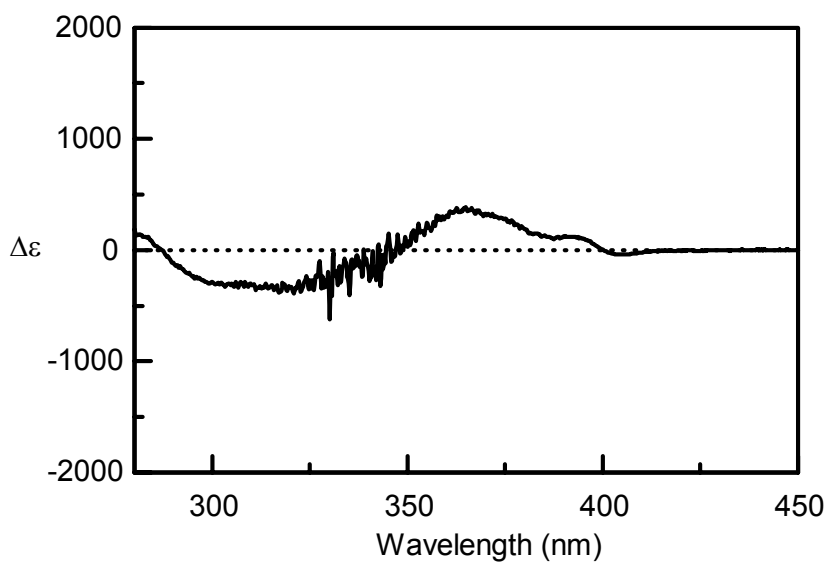

Figure S24. (a) Degree of aggregation of 7 by VPO (toluene, $40{ }^{\circ} \mathrm{C}$ ) at various concentrations after being heated at $40{ }^{\circ} \mathrm{C}$ for $3 \mathrm{~h}$. (b) $\mathrm{CD}$ spectrum $\left(1 \mathrm{mM}, 40{ }^{\circ} \mathrm{C}\right.$, toluene) of 7 after being heated at $40{ }^{\circ} \mathrm{C}$ for $3 \mathrm{~h}$. The same sample as that used in the VPO study at $1 \mathrm{mM}$ was used. 


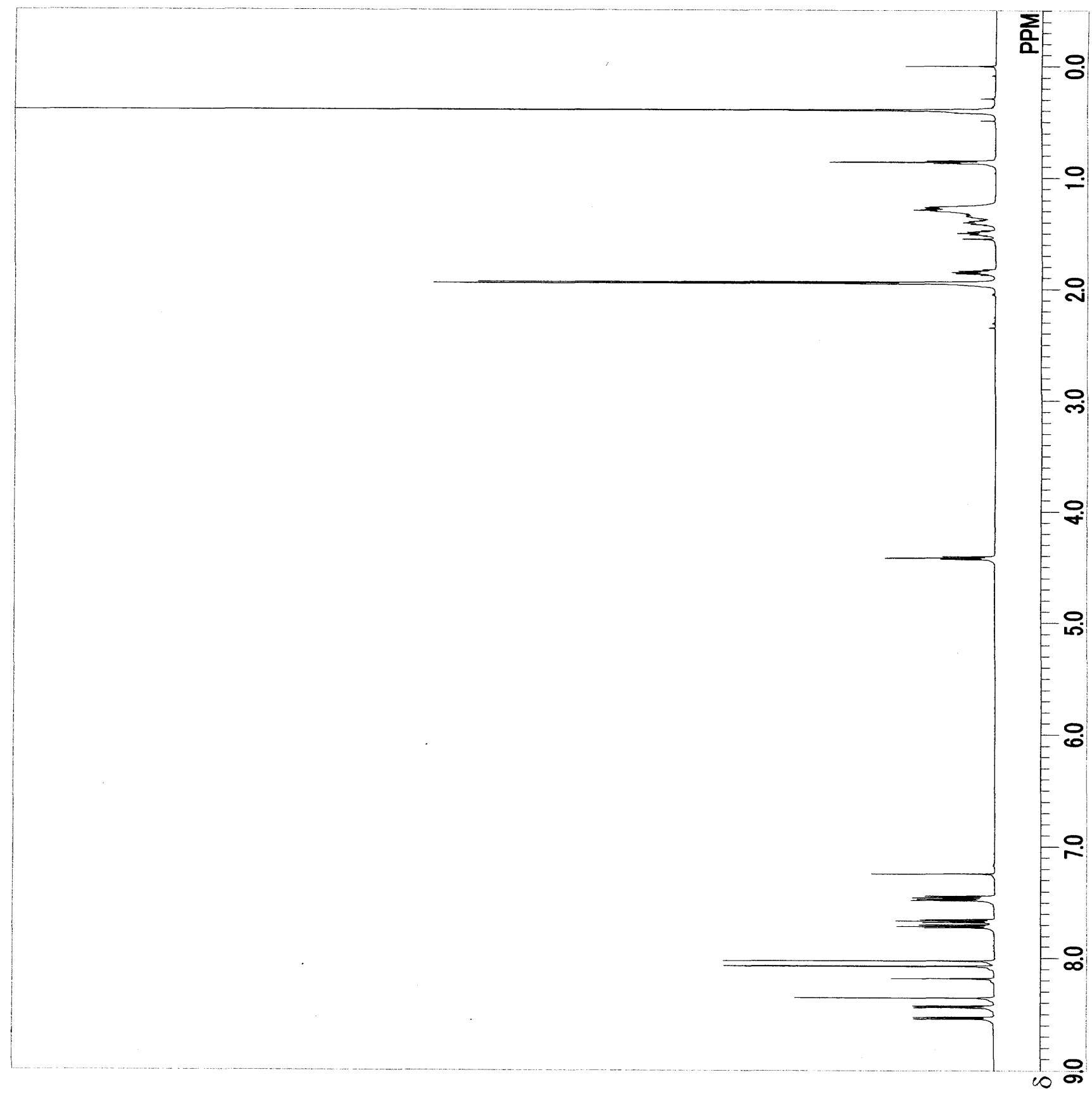

Figure S25. ${ }^{1} \mathrm{H}$ NMR $\left(600 \mathrm{MHz}, \mathrm{CDCl}_{3}, 25^{\circ} \mathrm{C}\right)$ spectrum of 2. 


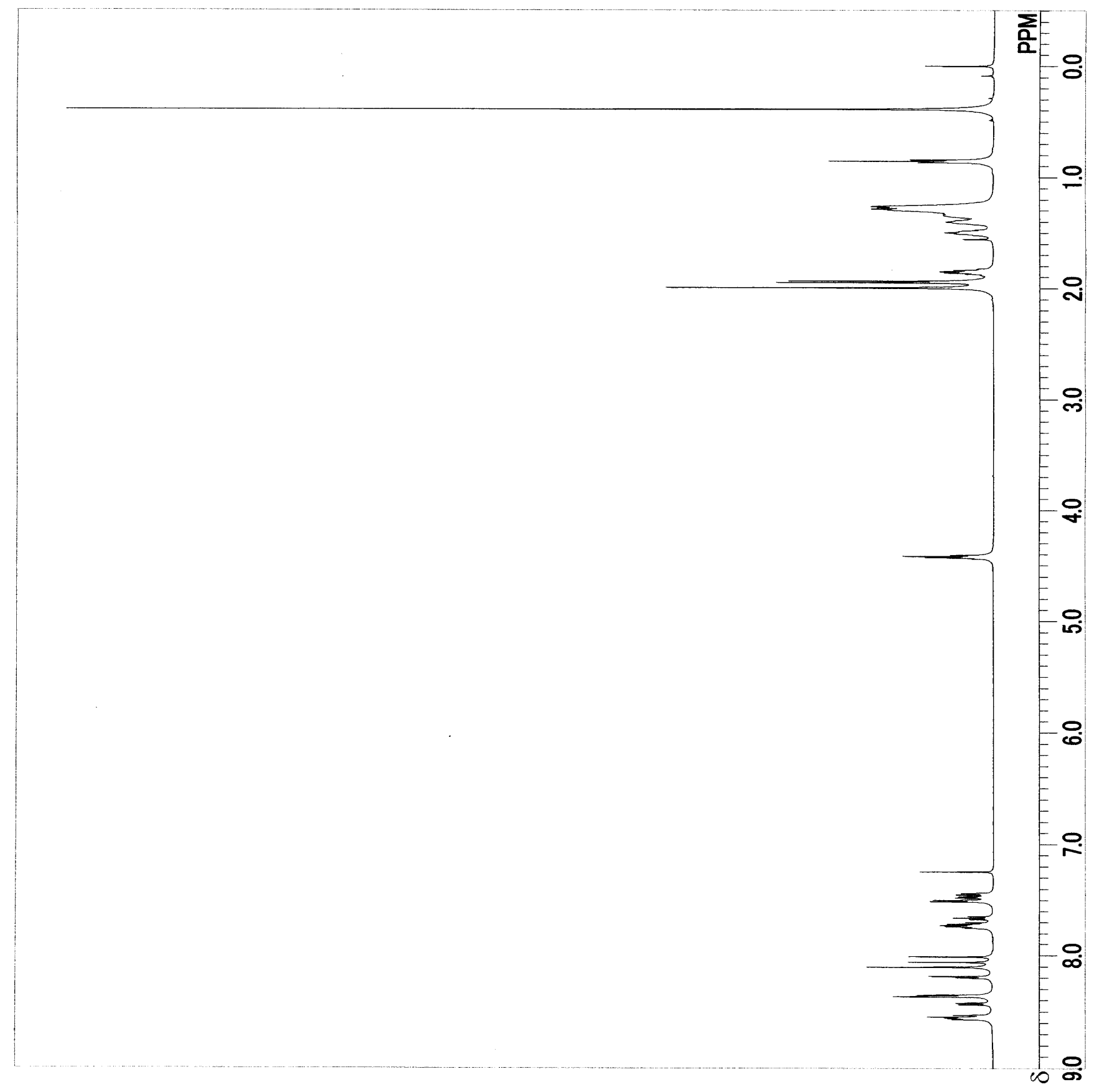

Figure S26. ${ }^{1} \mathrm{H}$ NMR $\left(600 \mathrm{MHz}, \mathrm{CDCl}_{3}, 25^{\circ} \mathrm{C}\right)$ spectrum of 4 . 


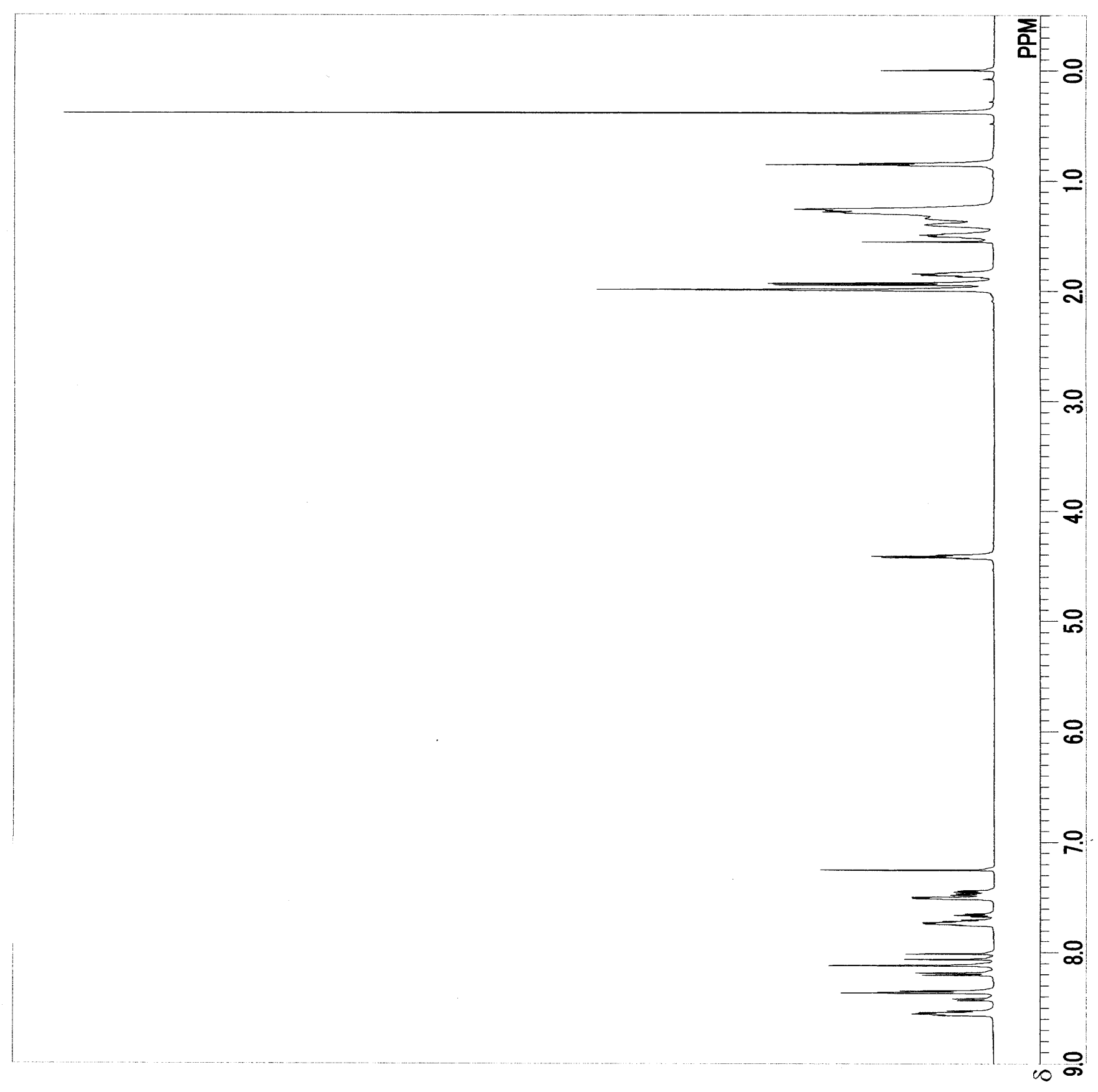

Figure S27. ${ }^{1} \mathrm{H}$ NMR $\left(600 \mathrm{MHz}, \mathrm{CDCl}_{3}, 25^{\circ} \mathrm{C}\right)$ spectrum of 5 . 


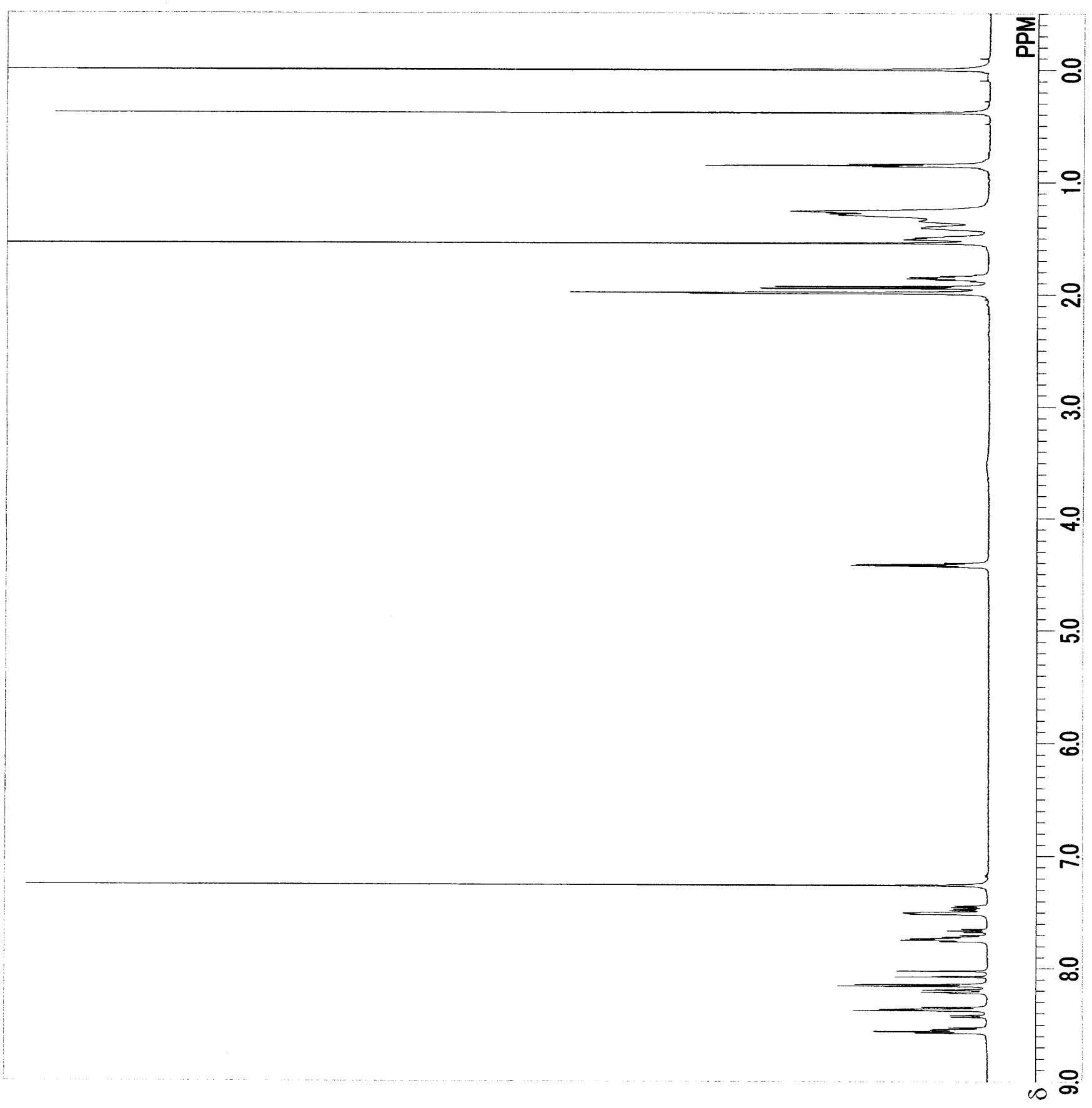

Figure S28. ${ }^{1} \mathrm{H}$ NMR $\left(600 \mathrm{MHz}, \mathrm{CDCl}_{3}, 25^{\circ} \mathrm{C}\right)$ spectrum of 6 . 


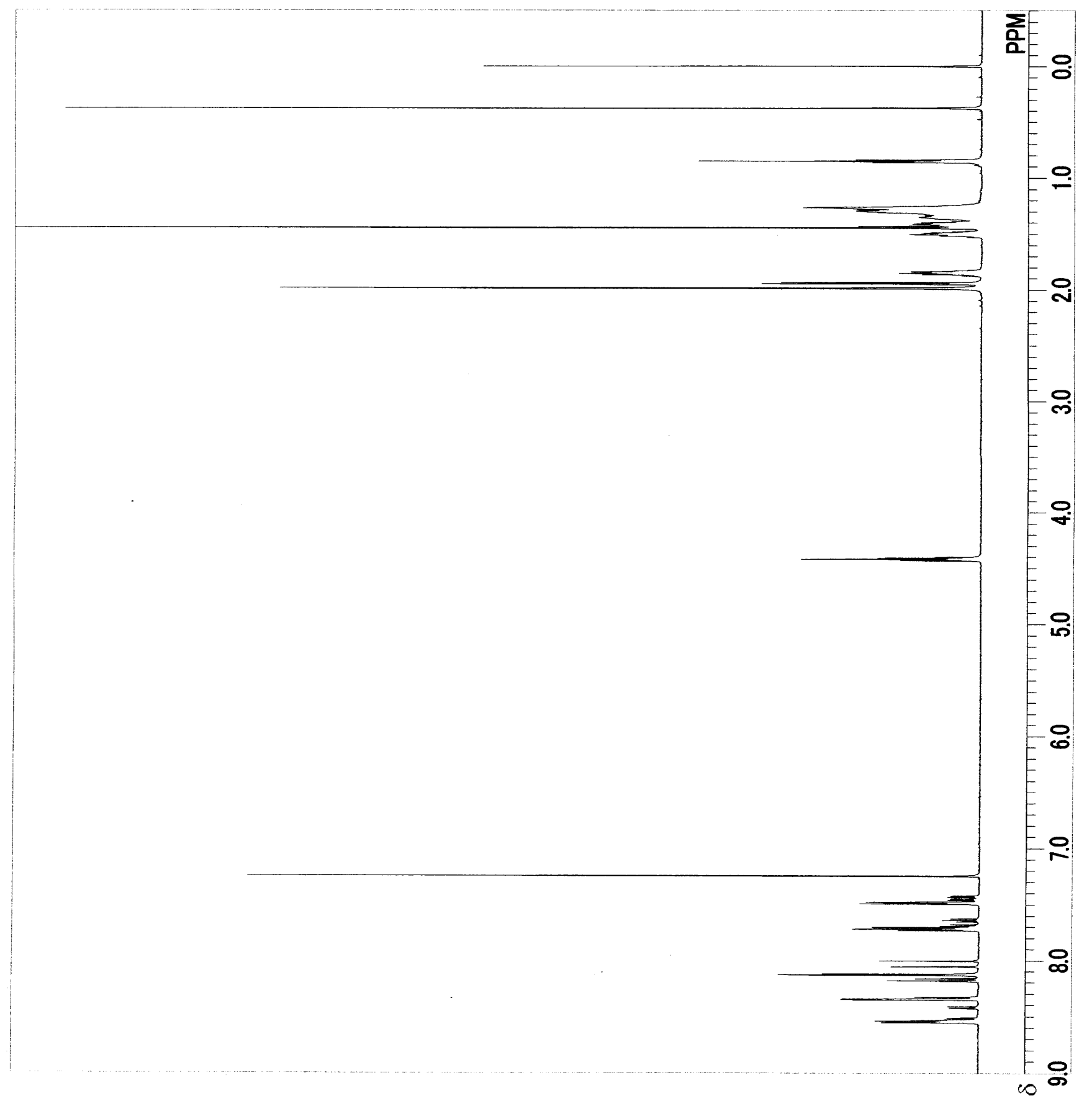

Figure S29. ${ }^{1} \mathrm{H}$ NMR $\left(600 \mathrm{MHz}, \mathrm{CDCl}_{3}, 1 \mathrm{mM}, 60^{\circ} \mathrm{C}\right)$ spectrum of 7 after being heated at $60{ }^{\circ} \mathrm{C}$ for $1 \mathrm{~h}$. 


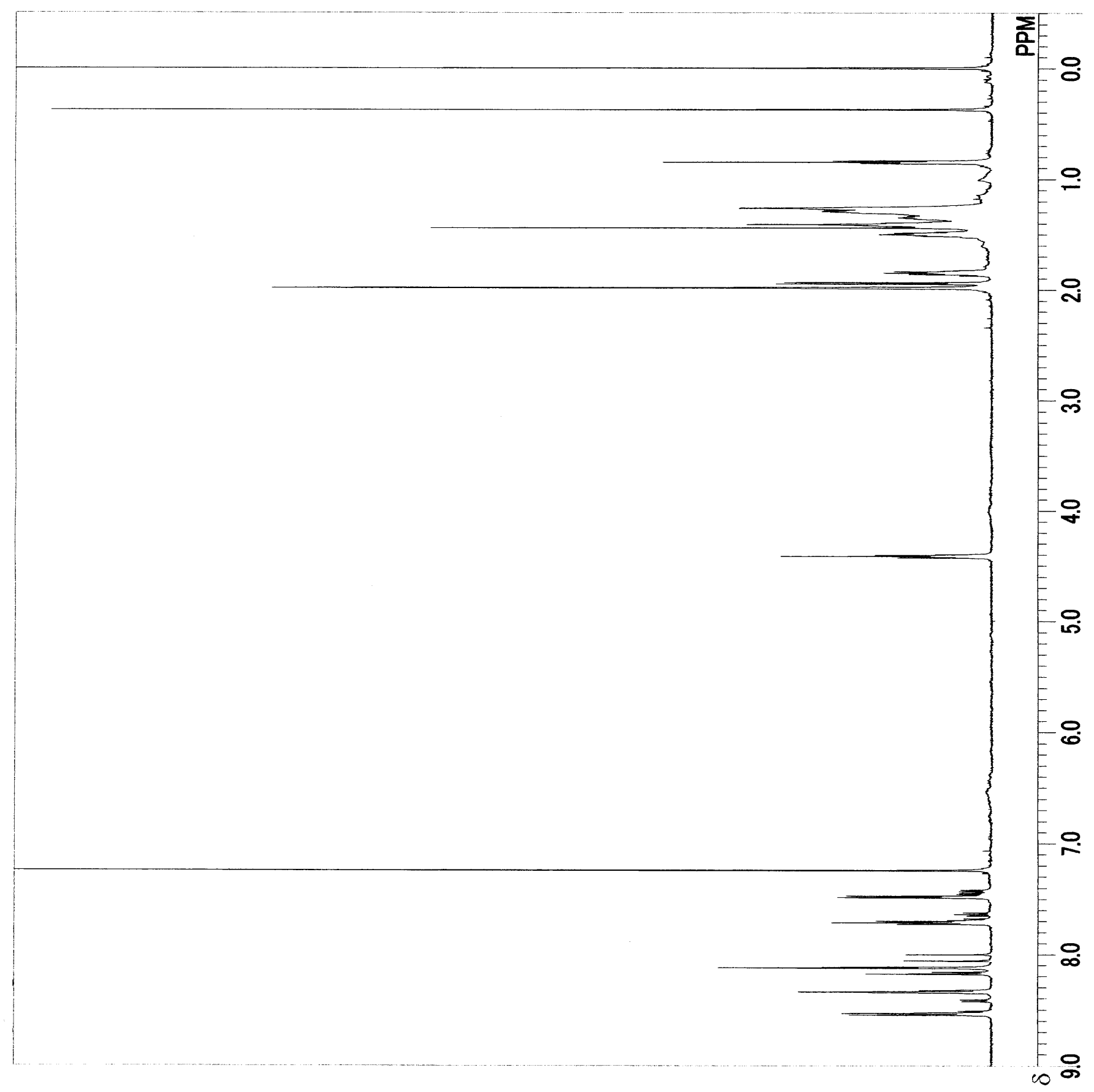

Figure S30. ${ }^{1} \mathrm{H}$ NMR $\left(600 \mathrm{MHz}, \mathrm{CDCl}_{3}, 1 \mathrm{mM}, 60^{\circ} \mathrm{C}\right)$ spectrum of 8 after being heated at $60^{\circ} \mathrm{C}$ for $2 \mathrm{~h}$. 


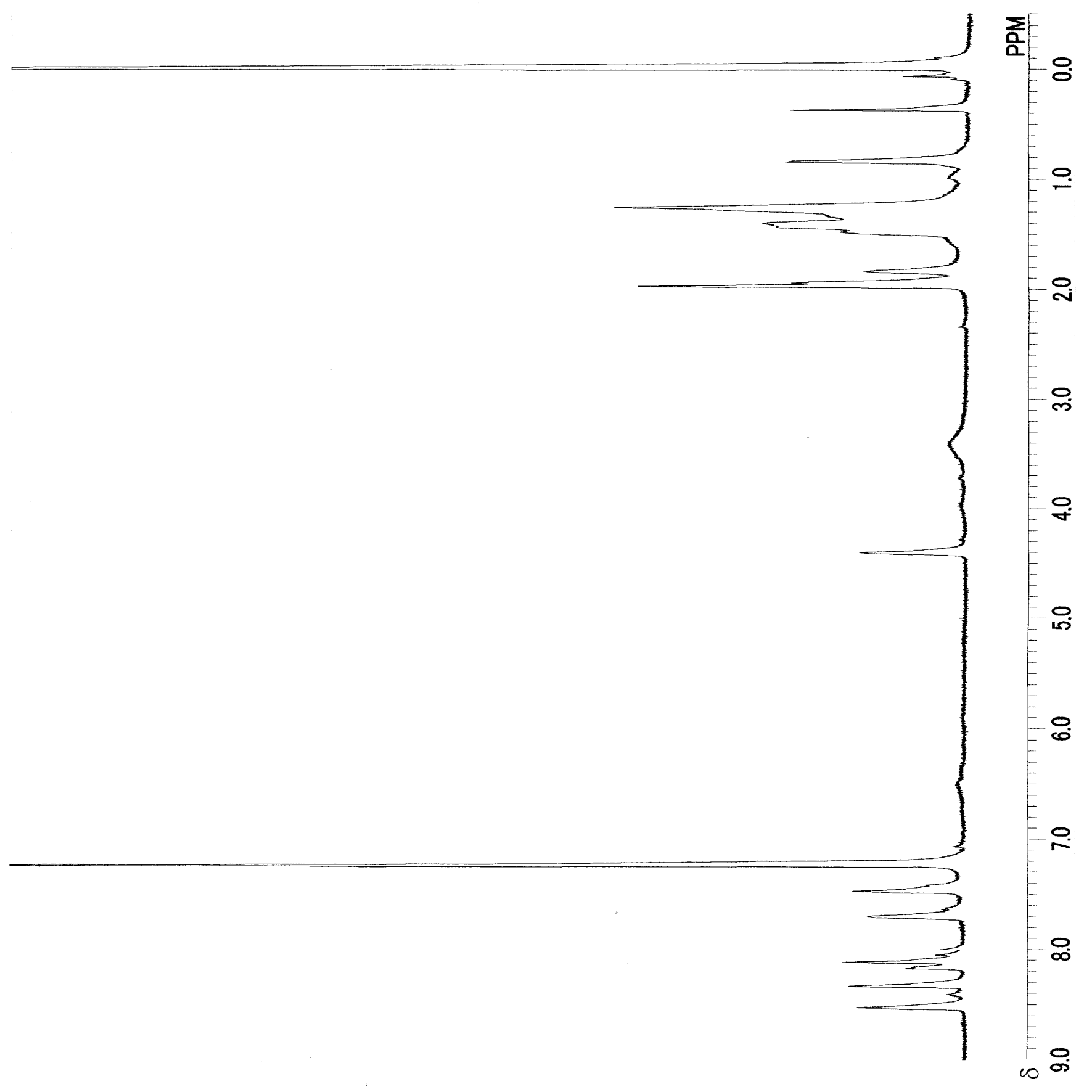

Figure S31. ${ }^{1} \mathrm{H}$ NMR $\left(600 \mathrm{MHz}, \mathrm{CDCl}_{3}, 0.20 \mathrm{mM}, 60^{\circ} \mathrm{C}\right)$ spectrum of 9 after being heated at $60{ }^{\circ} \mathrm{C}$ for $25 \mathrm{~h}$. 